\title{
Genetic differences between lung metastases and liver metastases from left-sided microsatellite stable colorectal cancer: next generation sequencing and clinical implications
}

\author{
Zhenghang Wang ${ }^{1}$, Xue Zheng ${ }^{2}$, Xicheng Wang ${ }^{1}$, Yawei Chen ${ }^{2}$, Zhongwu Li $^{3}$, Jianing Yu ${ }^{2}$, Wanning Yang ${ }^{2}$, \\ Beibei Mao ${ }^{2}$, Henghui Zhang ${ }^{2,4}$, Jian $\mathrm{Li}^{1}$, Lin Shen ${ }^{1}$ \\ ${ }^{1}$ Department of Gastrointestinal Oncology, Key Laboratory of Carcinogenesis and Translational Research (Ministry of Education), Peking University \\ Cancer Hospital \& Institute, Beijing, China; ${ }^{2}$ Genecast Biotechnology Co., Ltd., Wuxi, China; ${ }^{3}$ Department of Pathology, Key Laboratory of \\ Carcinogenesis and Translational Research (Ministry of Education), Peking University Cancer Hospital \& Institute, Beijing, China; ${ }^{4}$ Institute of \\ Infectious Diseases, Beijing Ditan Hospital, Capital Medical University, Beijing Key Laboratory of Emerging Infectious Diseases, Beijing, China \\ Contributions: (I) Conception and design: Z Wang, X Zheng; (II) Administrative support: L Shen, J Li; (III) Provision of study materials or patients: X \\ Zheng, X Wang, Y Chen, Z Li; (IV) Collection and assembly of data: Z Wang, J Yu, W Yang, B Mao, H Zhang; (V) Data analysis and interpretation: \\ All authors; (VI) Manuscript writing: All authors; (VII) Final approval of manuscript: All authors. \\ Correspondence to: Lin Shen, PhD, MD. Key Laboratory of Carcinogenesis and Translational Research (Ministry of Education), Peking University \\ Cancer Hospital \& Institute, Fucheng Road No. 52, Haidian District, Beijing, China. Email: shenlin@bjmu.edu.cn; Jian Li, PhD, MD. Key \\ Laboratory of Carcinogenesis and Translational Research (Ministry of Education), Peking University Cancer Hospital \& Institute, Fucheng Road \\ No. 52, Haidian District, Beijing, China. Email: oncogene@163.com.
}

Background: Data regarding the clinical characteristics and outcomes of lung metastases (LuM) from colorectal cancer (CRC) are different from those of liver metastases (LiM) from CRC. However, little is known about the genetic features of LuM. This study aimed to identify the different genetic characteristics of LuM and LiM from left-sided microsatellite stable CRC.

Methods: Tissue samples of the primary tumors and paired metastases from 18 CRC patients with isolated LuM (LuM cohort), 18 patients with isolated LiM (LiM cohort), and 10 locally advanced CRC patients without metastases (control cohort) were selected for next-generation sequencing. Patients in the LiM cohort had matched clinicopathological characteristics with the LuM cohort. The single-nucleotide variations (SNVs), copy number variations (CNVs), pathway alterations, and tumor mutation burdens (TMBs) were also calculated and analyzed.

Results: The CNV results showed that ZFHX4, GATA2, and FAM131B amplifications were more common in the metastatic cohorts than in the control cohort, while RECQL4 and FLCN amplifications were common in the controls. The LuM cohort had significantly higher proportions of $H N F 4 A, B R D 4$, and $U 2 A F 1$ amplification. The LuM, LiM, and control cohorts were successfully separated using pathway alteration analysis. The LuM cohort had more frequent alterations in the RTK/RAS pathway, HIPPO pathway, KRAS, and MET than the LiM group. The LuM cohort also had relatively higher TMBs than the LiM cohort.

Conclusions: CNVs in primary tumors could identify patients with LuM. Targeting the HIPPO pathway or MET and immune checkpoint inhibitors (ICIs) combined with other agents might be novel therapies for LuM.

Keywords: colorectal cancer (CRC); lung metastasis; liver metastasis; copy number variation (CNV); tumor mutation burden (TMB)

Submitted Mar 22, 2021. Accepted for publication Jun 15, 2021.

doi: $10.21037 / \mathrm{atm}-21-2221$

View this article at: https://dx.doi.org/10.21037/atm-21-2221

(c) Annals of Translational Medicine. All rights reserved. 


\section{Introduction}

Colorectal cancer (CRC) ranks as the third most commonly occurring cancer worldwide (1), while metastasis is the major cause of death in patients with cancer. The liver is one of the most frequent sites in which malignancies preferentially metastasize, followed by the lungs, lymph nodes, bone, and brain. Rectal cancers, especially middle and lower rectal cancers, tend to metastasize to the lungs and bones. While colon cancers mainly have a higher incidence of liver metastases (LiM) (2). Given that China has a much higher incidence of rectal cancer than Western countries (3), treating CRC patients with lung metastases $(\mathrm{LuM})$ is also an urgent health issue.

Patients with resected LiM could benefit from radical resection with perioperative treatments. However, most patients had unresectable LiM and should receive palliative pharmacotherapy as the standard care. It is currently recommended to apply the therapeutic strategy of LiM for patients with $\mathrm{LuM}$ due to the lack of specific guidelines for managing LuM CRC. Approximately only $10 \%$ of patients with initial LuM can undergo radical resection $(4,5)$ but they do not receive any benefits from adjuvant chemotherapy (6). Compared with $\operatorname{LiM}(7,8)$, the possibility of conversion from unresectable $\mathrm{LuM}$ to resectable $\mathrm{LuM}$ is relatively low $(5.7-7.1 \%)(5)$. Therefore, the standard treatment for most patients with LuM or LiM is pharmacotherapy, including chemotherapy and targeted agents. However, many metastatic CRC patients who failed to respond to standard therapies still have good Karnofsky performance status, yet no other drugs are available for use. This phenomenon is more obvious in CRC with LuM, since patients with $\mathrm{LuM}$ have longer survival duration and slower growth of cancer cells compared to LiM (9). The acquisition of the tumor genetic profiles may screen out specific targets of LuM. For instance, the frequency of HER2 positivity in LuM is $4 \%$, which is significantly higher than that in the primary tumor (1.8\%) (10). In the HERACLES and MyPathway trials, the promising antitumor activity of dual anti-HER2 therapy was observed $(11,12)$, indicating that HER2 might be a valuable therapeutic target of $\mathrm{LuM}$.

Limited data of the genetic features of LuM have been reported. A small case-match study has revealed that de novo mutations in LuM are different from those in LiM (13). A previous study has also demonstrated that KRAS and PIK3CA mutations are associated with the development of LuM (14). Taken together, the above evidence suggests that LuM may represent a unique molecular subtype from that of LiM in CRC patients. However, current findings are far from enough for a full elucidation of the genetic features of LuM compared to LiM. More biomarkers and targets are still needed in clinical practice. Therefore, this pilot study aimed to provide novel insights of genetic differences between LuM and LiM.

Some clinical and pathological characteristics, such as primary tumor location (left $v s$. right) and microsatellite instability (MSI) status (microsatellite stability $v s$. MSI), are associated with genetic profiles of metastatic CRC (15). This study focused on microsatellite stable (MSS) left-sided CRC which represented the most cases of CRC. To reduce other potential confounding factors that might influence the genetic features, we selected patients with isolated LuM, and matched them with isolated LiM patients according to the clinicopathological features, including age, sex, primary location, $\mathrm{T}$ stage, $\mathrm{N}$ stage, histology, and differentiation grade. As a result, the different genetic profiles between the LuM and LiM cohorts in this study could be considered as true molecular differences, while previous studies of genetic profiles in CRC did not establish these well-matched cohorts. By comparing the genetic profiles of primary tumors and the corresponding metastases from patients with $\mathrm{LuM}$ and LiM using next-generation sequencing (NGS), we found that the CNV characteristics of primary tumors can be used as a marker to distinguish LuM patients from LiM patients and patients without metastasis. The LuM cohort had more frequent alterations in the RTK/RAS pathway, HIPPO pathway, KRAS and MET than the LiM group. We present the following article in accordance with the MDAR reporting checklist (available at https://dx.doi. org/10.21037/atm-21-2221).

\section{Methods}

\section{Patients}

The clinical data of 213 patients with isolated LuM CRC who received treatment between 2008 and 2016 were retrospectively reviewed at the Department of Gastrointestinal Oncology, Peking University Cancer Hospital \& Institute. The main inclusion criteria of the LuM cohort were as follows: (I) had a biopsy or surgery of both the primary tumor and LuM; (II) both primary tumor and LuM tissue samples were available; (III) patients were diagnosed with left-sided CRC with isolated LuM. After excluding unqualified participants, a total of $18 \mathrm{LuM} \mathrm{CRC}$ patients were included in the LuM cohort. 


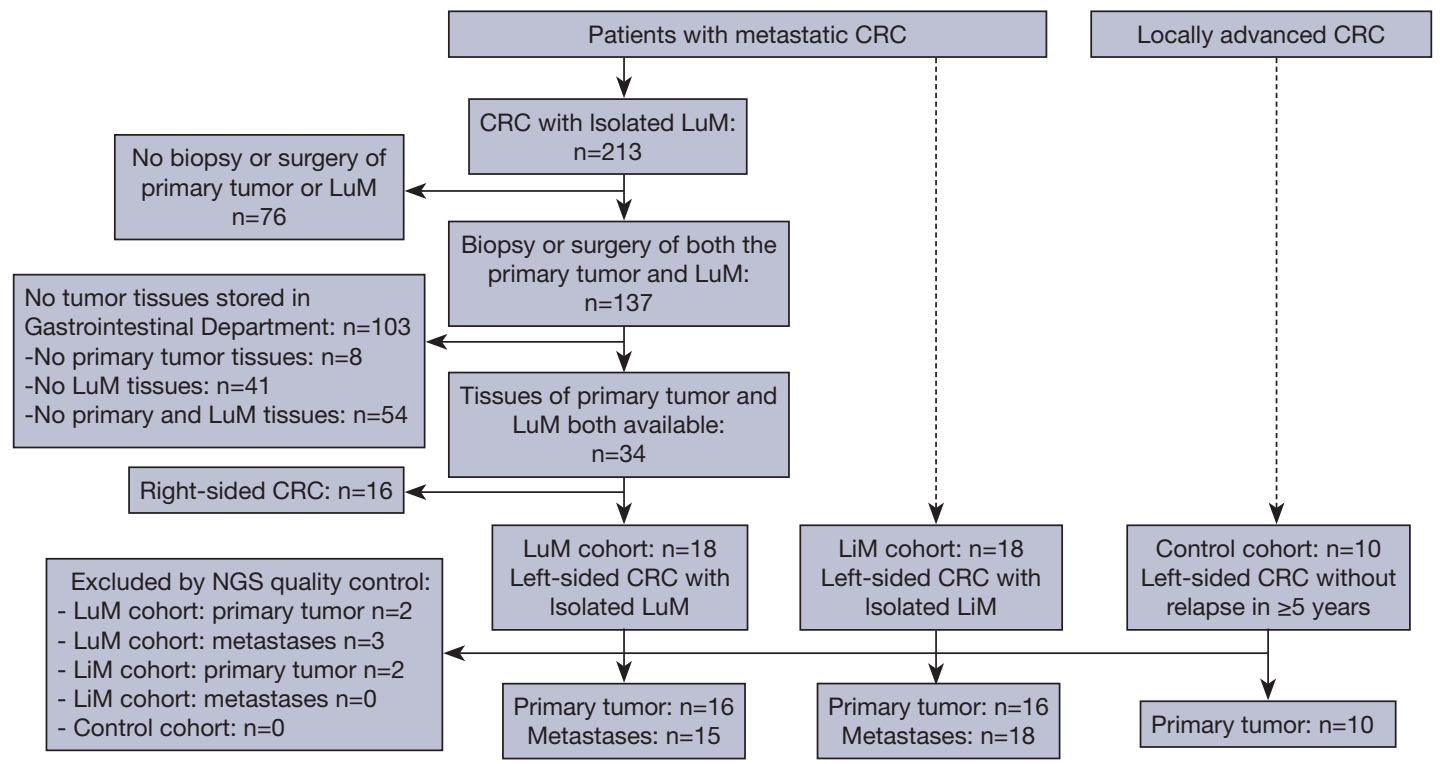

Figure 1 Flow diagram of patient selection. The LiM and LuM cohorts were matched 1:1 according to age (within the range of \pm 10 years, with the LuM patient as the reference), sex (male, female), primary location (left colon, upper rectum, middle rectum, and lower rectum), $\mathrm{T}$ stage (T1-2, T3-4), N stage (positive, negative), histology (with or without a mucinous carcinoma or signet ring cell carcinoma component), and differentiation grade (well to moderate, poor to undifferentiated). CRC, colorectal cancer; LuM, lung metastases; LiM, liver metastases; NGS, next-generation sequencing.

To investigate the different genetic characteristics between LuM and LiM, we also selected 18 patients with left-sided CRC with isolated LiM as the LiM cohort. The patients in the LiM cohort had matched clinicopathological characteristics as the $\mathrm{LuM}$ cohort to minimize bias in the study, including age, sex, primary location, T stage, $\mathrm{N}$ stage, histology, and differentiation grade. In addition, 10 patients with locally advanced CRC who underwent radical surgery without relapse within the last 5 years were also selected as the control cohort. The small sample size in the control cohort was due to the limited number of patients with locally advanced CRC admitted to our department. The detailed selection process is demonstrated in Figure 1. This study was conducted in accordance with the Declaration of Helsinki (as revised in 2013). This study was approved by the Beijing Cancer Hospital Ethics Committee (No. 2017KT91) and individual consent for this retrospective analysis was waived.

\section{Tissue samples}

Tissue samples from the LuM ( $\mathrm{n}=18)$ cohort, the LiM $(\mathrm{n}=18)$ cohort, and the control cohort $(n=10)$ were collected. Each sample had been stored for $2-5$ years prior to the study. In the metastatic cohorts (LuM and LiM cohorts), 23 out of the 36 primary tumor tissues had pre-operative treatment, while the other 13 had upfront resection after being diagnosed. Four of the $18 \mathrm{LuM}$ tissue samples received pre-operative treatment, whereas 17 of the $18 \mathrm{LiM}$ tissue samples had pre-operative treatment. All of the tissue samples from the control cohort received upfront resection.

\section{DNA extraction}

Genomic DNA was extracted from formalin-fixed paraffinembedded (FFPE) tumor tissues and fresh tissues soaked in preservation solution using a blackPREP FFPE DNA Kit (Analytik Jena, Germany) and a Tiangen Genomic DNA Kit (Tiangen, Beijing, PRC), respectively. The DNA concentration was measured using a Qubit dsDNA HS Assay Kit (Life Technologies, California, USA) following the manufacturer's protocol. The extracted DNA was stored at $-20{ }^{\circ} \mathrm{C}$ or directly interrupted.

\section{Targeted sequencing}

Genomic DNA was sheared into 150-200 bp fragments using a Covaris M220 Focused-ultrasonicator (Covaris, 


\section{Page 4 of 13}

Massachusetts, USA). Once the fragment size met the requirements, the KAPA HTP Library Preparation Kit (Illumina platform, KAPA Biosystems, Massachusetts, USA) was applied to construct the DNA Library according to the manufacturer's instructions. The DNA library was captured with a designed 1406-gene panel (Genecast, Beijing, China) that included major tumor-related genes. The captured samples were then subjected to a HiSeq X-Ten system (Illumina, San Diego, California, USA) for paired-end sequencing.

\section{Variant calling of single-nucleotide variations (SNVs) and insertions and deletions (indels)}

We used the Mutect2 tool from the Genome Analysis Toolkit (GATK v3.7) to collect somatic SNVs as well as insertions and deletions (indel) in tumor and normal samples. The NimbleDesign assay (1,406 genes) was used to identify mutations. The following filters were applied: (I) for mutations that were not in COSMIC (https://cancer. sanger.ac.uk/cosmic): mutant allele frequency $>3 \%$ and tumor variant frequency 5 times higher than normal tissue; for mutations in COSMIC and passed the Mutect2 filter: mutant allele frequency $>1 \%$ and tumor variant frequency 5 times higher than normal tissues; for mutations in COSMIC but did not pass the Mutect2 filter: mutant allele frequency $>1 \%$ and tumor variant frequency 10 times higher than normal tissues; (II) the number of mutant allele reads $>5$; (III) coverage $>50$; (IV) allele frequency $<20 \%$ using a group of healthy human plasma samples from the Genecast database ( $\mathrm{n}=30)$; (V) nonsynonymous SNVs and indels; (VI) located in exon regions; and (VII) allele frequency $<0.5 \%$ in the Exosome Aggregation Consortium (ExAC) (http://exac. broadinstitute.org) and allele frequency $<1 \%$ in the 1,000 Genomes Project (http://www.internationalgenome.org/ data/).

\section{Calling of copy number variations (CNVs)}

We used the CNVkit (v0.9.2) to obtain the $\log _{2}$ copy ratio from the tumor samples of each patient and each gene. A panel of healthy control blood samples was used for reference construction. A gene was defined as copy number gain $\left(\log _{2}\right.$ copy ratio $\left.<\log _{2} 1.5\right)$ or $\operatorname{loss}\left(\log _{2}\right.$ copy ratio $\left.<-\log _{2} 5 / 3\right)$ when the number of target intervals was $\geq 5$.
Wang et al. Genetic features of lung and liver metastases in CRC

\section{Tumor mutation burden (TMB) calculation}

For the determination of TMB, the number of somatic nonsynonymous SNVs (with depth $>100 x$ and allele frequency $\geq 2 \%$ ) detected with NGS (interrogating $\mathrm{Mb}$ of the genome) was quantified, and the value was extrapolated to the whole exome. Targeted sequencing was performed for TMB calculation. Alterations which are known to be bona fide oncogenic drivers were excluded. TMB was measured in mutations per $\mathrm{Mb}$.

\section{Hierarchical clustering and patbway alteration analysis}

We performed hierarchical clustering based on Euclidean distance using the heatmap package in $\mathrm{R}$ software. The corresponding gene mutations were extracted and the gene landscape was obtained using specific pathways, as described in previous research (15).

\section{Statistical analyses}

Statistical analyses were performed using $\mathrm{R}$ version 3.6.0, SPSS version 19.0 (SPSS, Inc., Chicago, IL, USA), and GraphPad Prism (version 7.00, La Jolla, CA, USA) software. Differences between proportions were evaluated using Fisher's exact test. The Kruskal-Wallis test was also applied to compare differences between multiple groups, while Dunn's multiple comparisons test was carried out to compare differences between 2 groups. All of the tests were two-sided, and $\mathrm{P}$ values less than 0.05 were regarded as statistically significant.

\section{Results}

\section{Clinicopathological characteristics}

A total of 46 participants were involved in this study, including 18 in the LuM cohort, 18 in the LiM cohort, and 10 in the control cohort. Two of the 18 primary tumors and 3 of the 18 metastatic lesions in the LuM cohort, and 2 of the 18 primary tumors in the LiM cohort failed to pass the quality control for NGS, and were therefore excluded from further genetic analysis. The clinicopathological characteristics of each cohort were collected and analyzed (Table 1). Approximately $60 \%$ of patients were male. The most common primary location of tumors was the rectum. The $\mathrm{T}$ stage was $\mathrm{T} 3-4$ in all cases, while the $\mathrm{N}$ stage was 


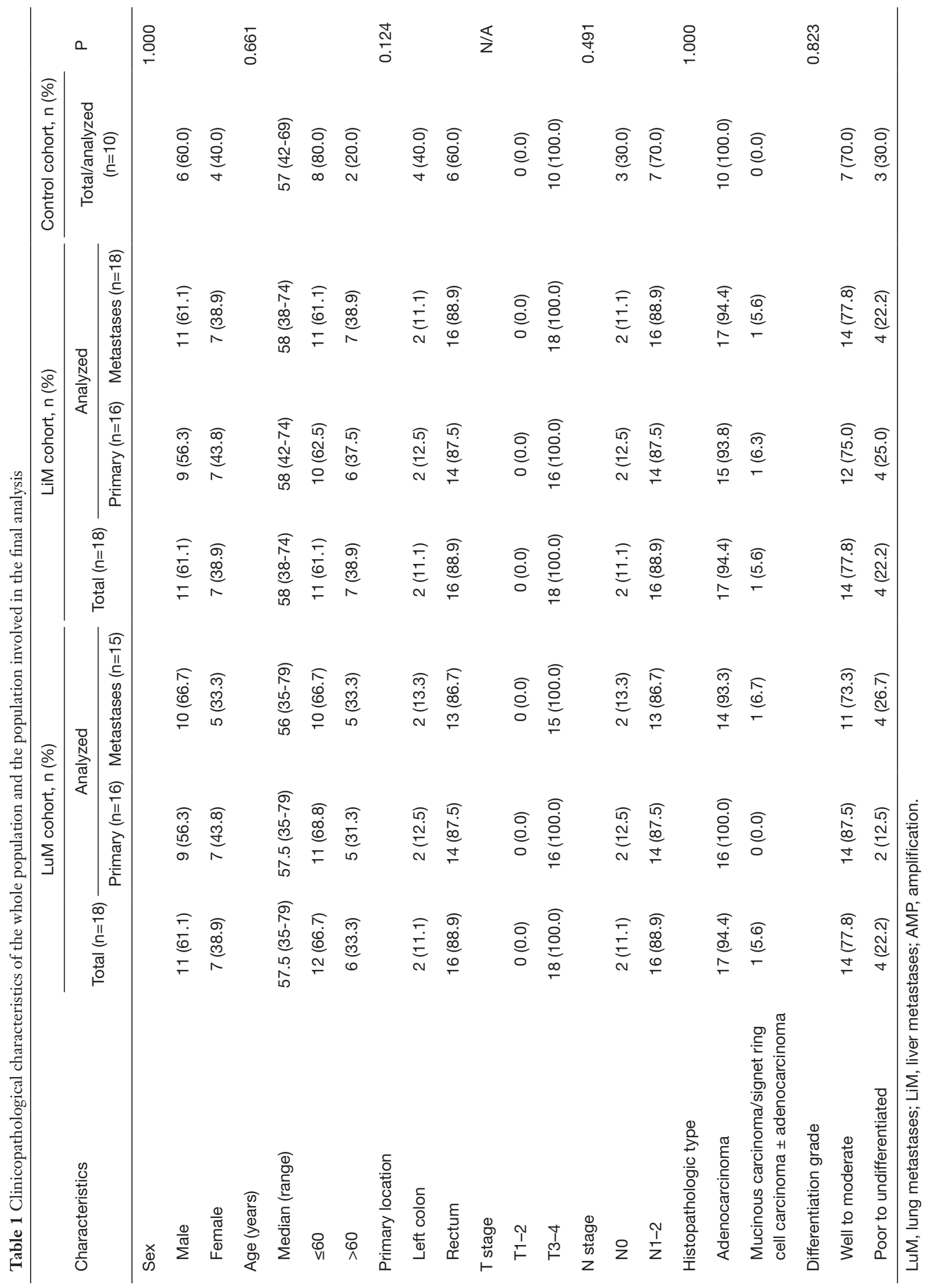


positive in most cases.

\section{Genetic landscapes of the primary tumors}

All primary tumors were MSS. The most common mutated gene was TP53 (69\%), followed by APC (64\%) and KRAS $(31 \%)$ in the whole population. No significant difference in SNVs was observed between the metastatic $(\mathrm{LuM}+\mathrm{LiM})$ cohort and the control cohort or between the LuM and LiM cohorts (Figure $2 A$, Table S1).

The results of CNVs showed that ZFHX4 (90.6\% vs. $10.0 \%, \mathrm{P}<0.001)$, GATA2 (34.4\% vs. $0.0 \%, \mathrm{P}=0.081)$, and FAM131B (34.4\% vs. $0.0 \%, \mathrm{P}=0.081)$ were commonly amplified in the metastatic cohort compared to the control cohort, while RECQL4 (6.3\% vs. $70.0 \%, \mathrm{P}<0.001)$ and FLCN (0.0\% vs. $30.0 \%, \mathrm{P}=0.010)$ were commonly amplified in the control cohort (Figure 2B, Table S2). Based on the CNV data of the 5 selected genes (FLCN, RECQL4, ZFHX4, GATA2, and FAM131B), hierarchical clustering of all the primary tumors revealed that the metastatic cohort and control cohort formed 2 separate clusters (Figure 2C).

The LuM cohort had significantly higher proportions of HNF4A (43.8\% vs. $0.0 \%, \mathrm{P}=0.007)$, BRD4 (50.0\% vs. $6.3 \%, \mathrm{P}=0.015)$, and $U 2 A F 1$ ( $31.3 \%$ vs. $0.0 \%, \mathrm{P}=0.043$ ) amplification than those in the LiM cohort (Figure $2 B$, Table S2). Based on the CNVs of 3 selected genes (HNF4A, $B R D 4$, and U2AF1) $(\mathrm{P}<0.05)$, hierarchical clustering of the primary tumors from the metastatic cohort demonstrated that 13 of the LuM patients and 2 of the LiM patients formed a cluster, while the remaining patients ( $3 \mathrm{LuM}$ patients and 14 LiM patients) formed another cluster (Figure 2D).

As patients with middle or lower rectal cancer are more likely to develop LuM than those with upper rectal cancer or left-sided colon cancer because of venous drainage (16), we further classified patients into a 'middle/lower rectum' group and a 'colon/upper rectum' group. The results indicated that the differences in gene amplification between the LuM and LiM cohorts in the middle/lower rectum group were comparable to those in the colon/upper rectum group (Figure S1).

\section{Pathway alteration analysis}

The landscapes of 10 selected pathways and their frequencies, including WNT, TGF $\beta$, RTK/RAS, PI3K, P53, NRF2, NOTCH, MYC, HIPPO, and cell cycle, in the primary and metastatic lesions of all cohorts are demonstrated in Figure 3 and Table S3. In all of the specimens involving primary and metastatic tumors, the 3 most altered pathways were RTK/RAS (80.0\%), P53 (76.0\%), and WNT (68.0\%), while the alteration frequency of the other pathways ranged from $2.6 \%$ (the NRF2 pathway) to $42.1 \%$ (the NOTCH pathway).

The analysis of pathway alterations in the primary tumors showed that the LuM cohort had a higher rate $(87.5 \%)$ of RTK/RAS pathway alterations than the LiM $(68.8 \%)$ and control (70\%) cohorts, with no significant differences. PI3K pathway alterations were more common (50.0\%) in the LuM cohort with no statistical significance. The rest of the pathways had similar alterations in the 3 cohorts (Figure 3, Table S3).

Regarding metastatic tumors, LuM was significantly associated with RTK/RAS pathway alterations $(100 \%$ vs. $72.2 \%, \mathrm{P}=0.049)$ compared with LiM. Furthermore, the P53 pathway (93.3\% vs. 66.7\%, $\mathrm{P}=0.095)$ and the HIPPO pathway $(33.3 \%$ vs. $5.6 \%, \mathrm{P}=0.070)$ showed more frequent alterations in the LuM cohort than in the LiM cohort (Figure 3, Table S3).

In the RTK/RAS pathway of the LuM cohort, the alteration frequency of $K R A S$ was the most common (73.3\%) one, which was markedly higher than that in the LiM cohort (38.9\%, $\mathrm{P}=0.080$ ). In the primary tumors of the LuM cohort, the $K R A S$ alteration frequency was $43.8 \%$, which was slightly higher than the LiM (31.3\%) and control (30.0\%) cohorts. The MET alteration rate was $40.0 \%$ and $5.6 \%$ in $\mathrm{LuM}$ and LiM, respectively $(\mathrm{P}=0.030)$, whereas there were no MET alterations in the primary tumors (Figure 3).

\section{Genetic characteristics associated with immune checkpoint inbibitors (ICIs)}

The median TMB of the primary tumors in the LuM cohort was $9.518 \mathrm{SNV} / \mathrm{Mb}$, which was significantly higher than that in the LiM (1.003 SNVs/Mb, $\mathrm{P}=0.003)$ and control (3.987 SNVs/Mb, $\mathrm{P}=0.030$ ) cohorts. Meanwhile, the LuM cohort demonstrated notably higher TMB levels than the LiM cohort (6.022 vs. $2.02 \mathrm{SNV} / \mathrm{Mb}, \mathrm{P}=0.044)$ (Figure 4, Table 2). A TMB $>10 \mathrm{SNV} / \mathrm{Mb}$ was observed more frequently in samples from the LuM cohort than the other 2 cohorts $(\mathrm{P}=0.004)$ (Table 2$)$.

The panel used in this study contained several genes whose alterations might contribute to the response (positive gene) or resistance (negative gene) to ICIs. The alteration frequencies of these positive and negative genes were not different between the primary and metastatic lesions or between different cohorts (Figure 5, Table S4). 

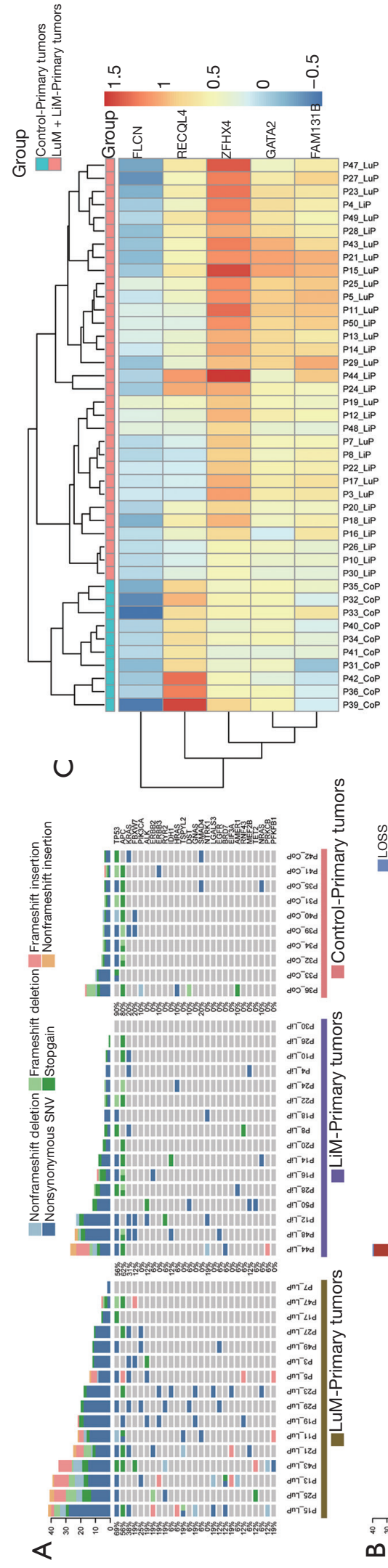

क

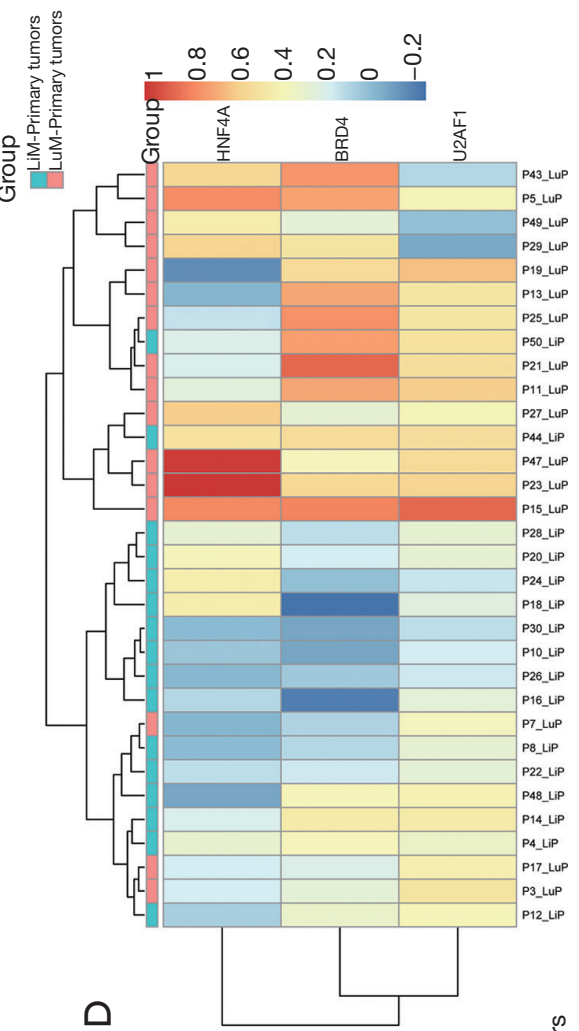

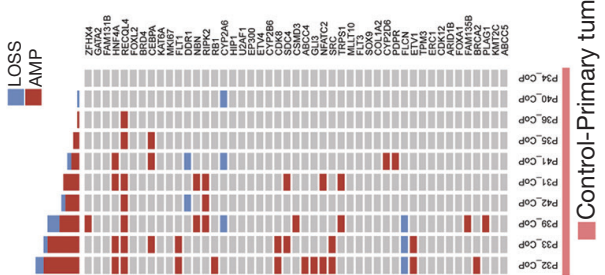

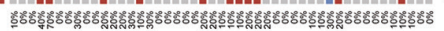

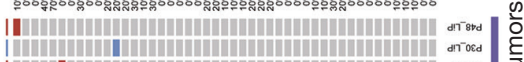
1 I | |

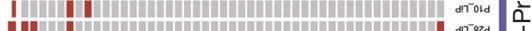
H PI - |

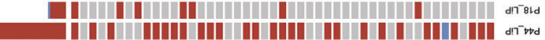

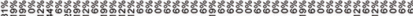

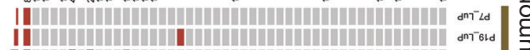
' H| -

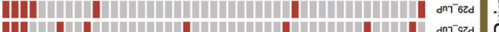
$\mathrm{H}$ 期

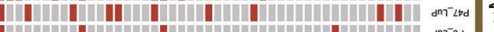

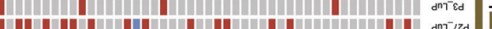
Н Н Н |Н НН Н Н |

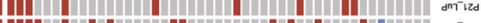
H | |

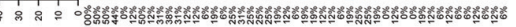

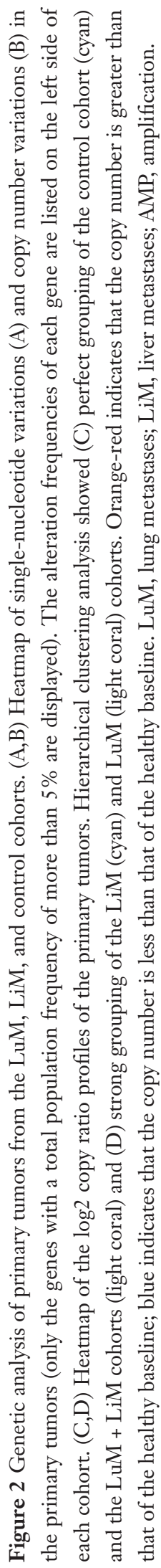



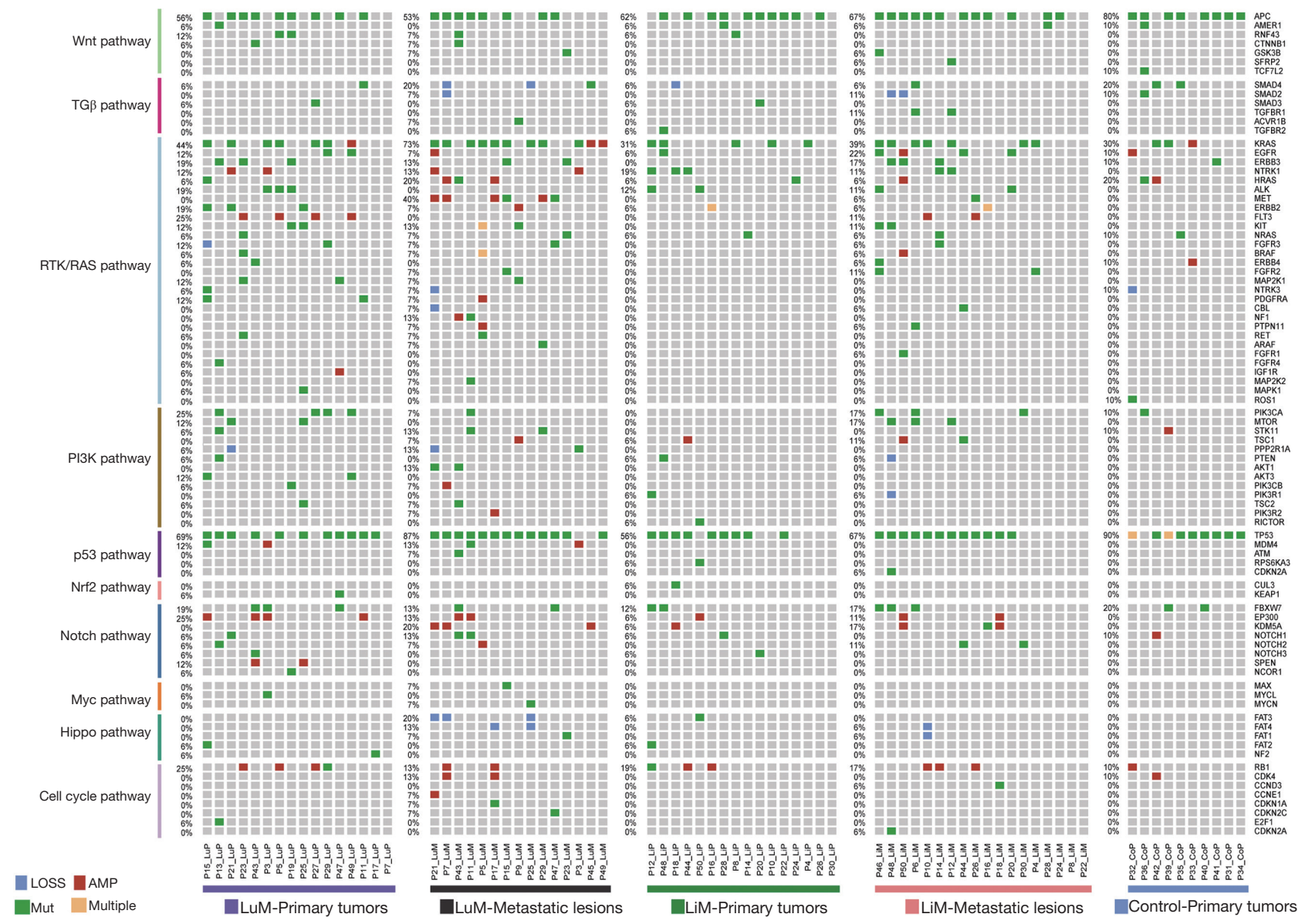

Figure 3 Alteration frequencies per gene in each pathway in the primary tumors and metastatic lesions. Blue: loss of copy number; red: AMP; green: SNV and/or indel; orange: concurrent variation (SNV, indel, or CNV). LuM, lung metastases; LiM, liver metastases; AMP, amplification; Mut, mutation; SNV, single-nucleotide variation; CNV, copy number variation.

\section{Discussion}

In this study, all primary tumors and metastatic lesions were MSS, indicating the high concordance of MSI status between primary tumors and metastases, which was in accordance with previous findings (17). Therefore, the population in this study was relatively homogeneous. Considering other clinical and pathological characteristics were also matched between LuM and LiM cohorts, it was believed that the different genetic profiles of LuM and LiM were relatively credible despite of the small sample number involved.

Early identification of locally advanced CRC patients who have a high risk of developing LuM can help with postoperative surveillance. Several clinical studies have shown that LuM is associated with $R A S$ mutation (18-21), PIK3CA mutation (22), and MTDH amplification (23).
However, the current findings regarding these gene alterations were not sufficient to predict LuM development. A previous study demonstrated that $45.3 \%$ of patients with KRAS mutations developed LuM, while $37.3 \%$ of patients developed LiM (19). Our results are consistent with the previous findings in that 13 patients had KRAS mutant primary tumors, 6 patients developed LuM, and 5 patients developed LiM. In 29 patients with KRAS wild-type primary tumors, 10 and 11 patients developed LuM and LiM, respectively.

We also compared the genetic profiles of primary tumors from the LuM cohort, the LiM cohort, and the control cohort to explore the 'metastatic signatures' of CRC using NGS. The results demonstrated that the amplifications of $Z F H X 4, G A T A 2$, and FAM31B were common in the metastatic cohorts, while frequent amplifications of FLCN 
and RECQL4 were observed in the control cohort. Zinc Finger Homeobox 4 (ZFHX4), a putative transcription factor, plays a crucial role in regulating glioblastoma pathogenesis (24). In the CRC cell line HCT-116, which has high expression of ZFHX4, the cell-cell junctions become closer. In contrast, the down-regulation of ZFHX4 could inhibit the capacity for cell migration. Furthermore, patients with high ZFHX4 mRNA levels are reported to have a poorer prognosis compared to those with low mRNA levels (25). GATA binding protein 2 (GATA2), a key member of the zinc finger transcription factor family, contributes to the development of hematopoietic malignant disorders and solid tumors, such as breast cancer and nonsmall cell lung cancer. High GATA2 expression is associated with later clinical stage of CRC (26). In our study, GATA2 and $Z H F X 4$ were amplified more frequently in metastatic CRC than in locally advanced CRC, indicating that these

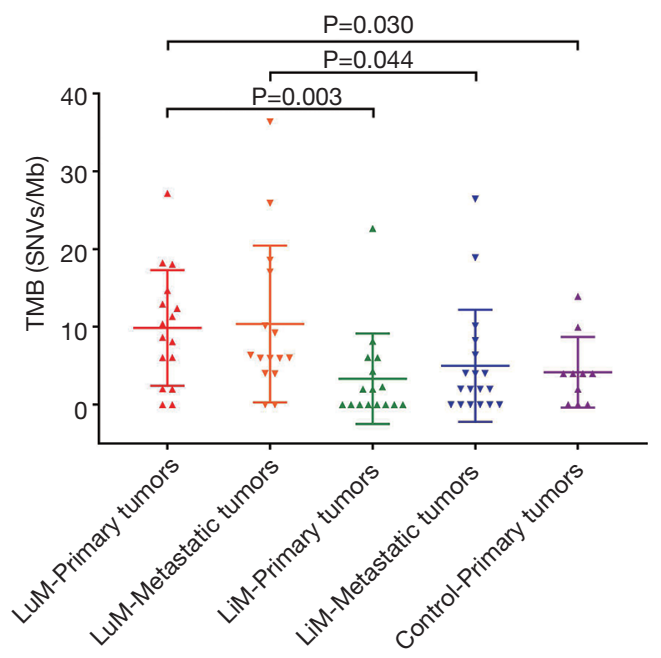

Figure 4 Tumor mutation burdens of the primary tumors and metastatic lesions. Data are mean \pm SD. SNV, single-nucleotide variation; LuM, lung metastases; LiM, liver metastases; SD, standard deviation. genes might have important roles in tumor invasion and metastasis. Given that both GATA2 and ZHFX4 genes encode transcription factors, the regulation of transcription factors might be a potential metastatic mechanism of CRC by either activating oncogenes or inactivating suppressor genes. Further studies are required to validate the association of mRNA levels or protein expression with gene amplification, and to explore detailed metastatic mechanisms. RECQL4, one of the RECQ helicases, is responsible for unwinding double-stranded nucleic acids and maintaining genomic integrity. CRC patients had significantly higher mRNA levels of RECQL4 than normal colonic mucosa, which suggests the potential survival advantage of CRC cells over normal tissues (27). In our study, RECQL4 amplification was detected in 7 out of 10 controls, while only 2 out of 32 metastatic patients had RECQL4 amplification. We found that most primary tumors without metastases need RECQL4 amplification to remain tumorigenic, while those with metastatic potential do not.

The results of hierarchical clustering analysis showed that patients with either $\mathrm{LuM}$ or LiM could be separated from those with locally advanced CRC in accordance with the CNVs of the above 5 genes, which raised the possibility of establishing a 'metastatic signature' of CRC.

Three additional genes, BRD4, HNF4A, and U2AF1, showed significantly higher amplification frequency in the LuM cohort than in the LiM cohort. BRD4 is an epigenetic regulator that localizes to DNA by binding to acetylated histones and plays an important role in the pathogenesis of CRC $(28,29)$. Preclinical work has demonstrated that $B R D 4$ inhibitors could inhibit tumor migration, invasion, and distal metastases (29). The transcription factor $\mathrm{HNF} 4 \alpha$, a member of the superfamily of nuclear receptors, has a pivotal role in oncogenic metabolism depending on the tissue specificity (30). The inhibition of tumor growth and proliferation in CRC after HNF4 $\alpha$ suppression indicates that $H N F 4 A$ is an oncogene (31). In the present study, $B R D 4$ and $H N F 4 A$ amplification was observed in $50 \%$ and

Table 2 Tumor mutation burdens of primary and metastatic lesions in LuM, LiM and Control Cohorts

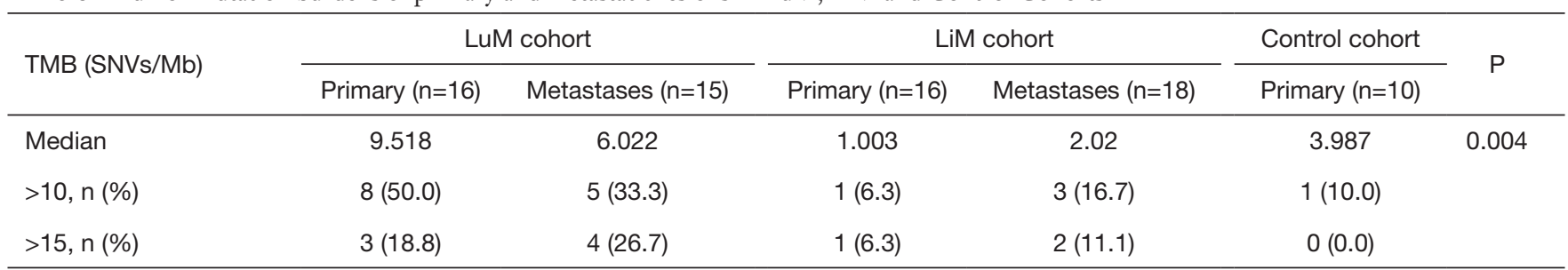

LuM, lung metastases; LiM, liver metastases. 

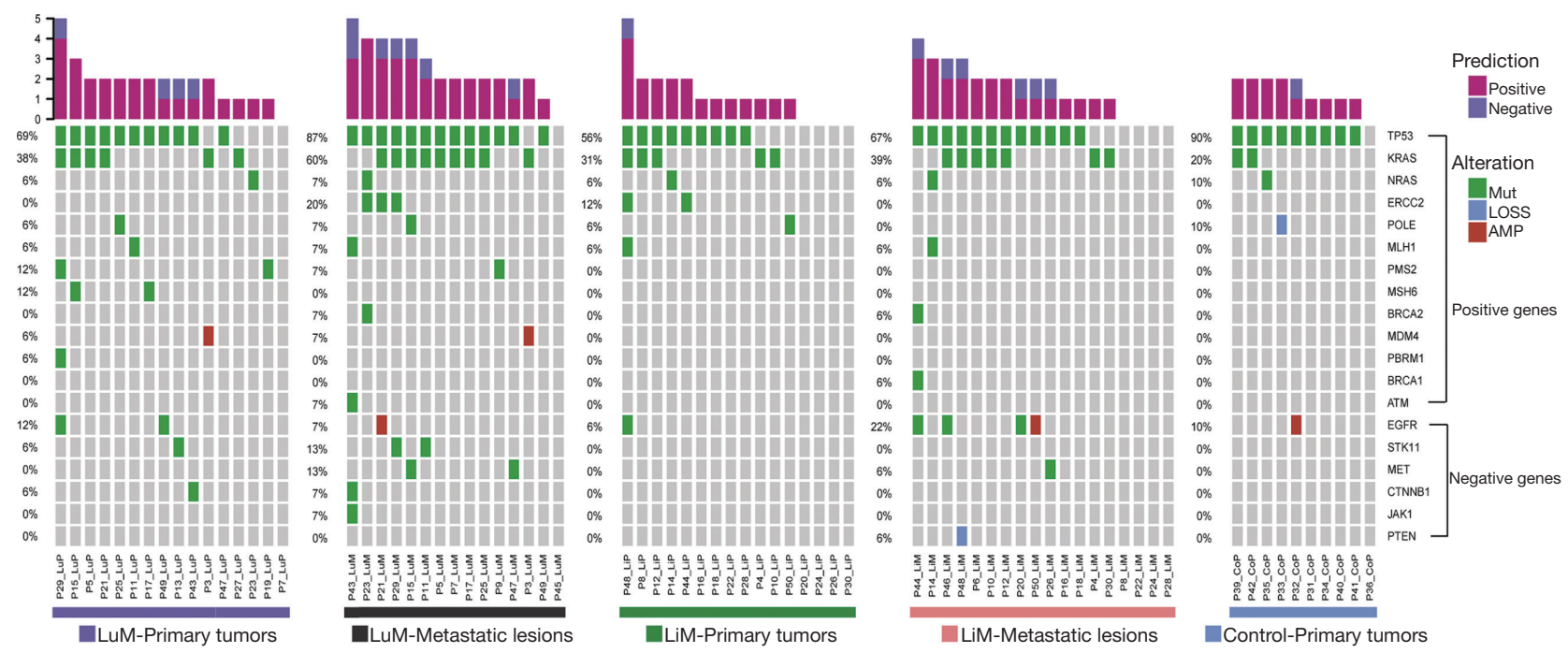

Figure 5 Gene alterations in the primary tumors and metastatic lesions probably associated with ICIs. "Positive" and "negative" genes indicate that the corresponding genes are associated with good and poor clinical outcomes, respectively, following treatment with ICIs. LuM, lung metastases; LiM, liver metastases; AMP, amplification; Mut, mutation; ICIs, immune checkpoint inhibitors.

$43.8 \%$ of patients with $\mathrm{LuM}$, respectively. Therefore, $B R D 4$ and $H N F 4 A$ inhibitors may be able to reduce the risk of developing LuM.

Moreover, we found that 8 out of 9 patients with $B R D 4$ amplification, 7 patients with $H N F 4 A$ amplification, and 5 patients with $U 2 A F 1$ amplification in the primary tumors developed LuM. Based on the CNV alterations specific to $\mathrm{LuM}$, cases with $\mathrm{LuM}$ were separated from those with $\mathrm{LiM}$ in the hierarchical clustering. However, these findings should be further validated in an independent cohort with a large number of samples.

At present, pharmacotherapy is the major treatment strategy for metastatic tumors. Although LuM has better survival than most distal metastases (9), there are no effective drugs for patients with LuM. In this genetic analysis, we aimed to explore the possibility of new therapeutic targets and ICIs for patients with LuM. The comparison of pathway alterations between LuM and LiM demonstrated that the RTK/RAS pathway and KRAS alterations were enriched in the LuM cohort, indicating the ineffectiveness of anti-EGFR antibodies. Additionally, MET amplification and the activation of the HIPPO pathway which were common in LuM may serve as therapeutic targets for LuM.

The MET gene, an important oncogene, encodes the tyrosine kinase receptor for hepatocyte growth factor and promotes tumor growth, invasion, and metastasis (32).
It is located upstream of the KRAS gene in the RTK/ RAS pathway (15), while its amplification is an acquired mechanism of resistance to EGFR inhibitors (33). MET amplification was observed in approximately $1 \%$ of untreated metastatic CRC (mCRC) (33) and 1\% of leftsided mCRC (34). Notably, none of the patients received anti-EGFR therapy in our LuM cohort, however, MET amplification was observed in 4 out of $16(25 \%) \mathrm{LuM}$ cases, indicating a potential role for MET inhibition in the therapeutic strategy of LuM.

In a multicenter phase 2 study, 41 anti-EGFRresistant patients with high MET expression and wildtype KRAS mCRC received the MET inhibitor tivantinib plus cetuximab, which showed a $9.8 \%$ overall response rate (ORR) and $43.9 \%$ disease control rate. In 13 tested patients with $M E T$ amplification, 2 patients with $M E T$ amplification achieved partial response (PR), while only 1 of the 11 patients without MET amplification achieved PR (32). Therefore, anti-MET therapy might be suitable for MET-amplified LuM. Nevertheless, it should be noted that all cases of MET amplification in this study were accompanied by KRAS mutations (Figure 3). Consequently, the combination of MET inhibitors and KRAS inhibitors, such as AMG 510 (35), might become a novel therapeutic target for LuM.

The HIPPO pathway is a cancer signaling network (36), and its alteration has been observed in approximately $10 \%$ 
of MSS CRCs without POLE mutations (15). Here, the mutation or loss of copy numbers of tumor suppressor genes including FAT1, FAT2, FAT3, and FAT4 in the HIPPO pathway were found in $33.3 \%$ of LuM, indicating that the HIPPO pathway might be activated and that a HIPPO pathway inhibitor might have a potential role in the treatment of LuM.

ICIs are not effective in $95 \%$ of patients with MSS mCRC (34), making it urgent to find new immunotherapies. Recently, durvalumab plus tremelimumab had better overall survival (OS) compared to placebo in MSS patients with a TMB $>28 \mathrm{SNVs} / \mathrm{Mb}$ (21\% of MSS patients) (37). Our results showed that patients with $\mathrm{LuM}$ were more likely to have higher TMB levels than patients with LiM, suggesting that patients with LuM may be a target population to receive combined ICIs. In the phase 1 trial REGONIVO, regorafenib plus nivolumab resulted in a $33 \%$ ORR in patients with MSS CRC (38). More recent data has shown that patients with $\mathrm{LuM}$ could receive more benefit from this treatment regimen than those with LiM in MSS CRC (39). These interesting findings might be explained partially by the relatively high TMB in LuM and the unexplored microenvironment in different metastatic organs. However, further analyses are required to find out if CRC patients with LuM could be the potential treatment population of ICIs.

This study has some limitations. First, the sample size was relatively small with minor statistical power due to the strict inclusion criteria, which allowed the close association of genetic differences with the metastatic phenotypes among different cohorts. Further studies with larger sample sizes in an independent cohort are essential to validate our findings. Second, most genetic differences were discovered as copy number alterations using NGS. These CNVs were not validated by RNA analysis or immunohistochemistry due to the lack of additional specimens. This is because the LuM and LiM specimens were collected prior to this study. Third, this pilot study showed the potential genetic differences and different amplification frequencies of certain genes between LuM and LiM, such as ZFHX4 and BRD4. However, their detailed functions and affected pathways related to specific metastatic organs remain to be investigated, and our data are currently available only at the DNA level, with a lack of exploration at the RNA level and epigenetic level. Lastly, the results were obtained from left-sided MSS CRC, and could not be extrapolated to right-sided CRC. Right-sided CRC with LiM had a poorer prognosis compared to left-sided CRC (40), while those with LuM seemed to have similar survivals regardless of primary location (5). Therefore, the underlying genetic differences between LuM and LiM in right-sided CRC should be further investigated.

\section{Conclusions}

This study presented evidence that the genetic characteristics of LuM from left-sided MSS CRC were distinct from those of LiM from left-sided MSS CRC. Patients with LuM could be separated from those with $\mathrm{LiM}$ and without metastases based on CNV features of the primary tumors. BRD4 and $H N F 4 A$ inhibitors might reduce the risk of developing LuM. For LuM patients with MSS CRC, targeting the HIPPO pathway or MET alterations might become a novel approach, and ICIs combined with other agents need to be investigated, especially for patients with high TMB.

\section{Acknowledgments}

We thank American Journal Experts (https://www.aje. $\mathrm{cn} /$ ) for linguistic assistance during the preparation of this manuscript.

Funding: This work was supported by the National Key Sci-Tech Special Project of China (grant number 2018ZX10302207).

\section{Footnote}

Reporting Checklist: The authors have completed the MDAR reporting checklist. Available at https://dx.doi. org/10.21037/atm-21-2221

Data Sharing Statement: Available at https://dx.doi. org/10.21037/atm-21-2221

Conflicts of Interest: All authors have completed the ICMJE uniform disclosure form (available at Available at https:// dx.doi.org/10.21037/atm-21-2221). All authors report funding from the National Key Sci-Tech Special Project of China (grant number 2018ZX10302207). XZ, YC, JY, WY, $\mathrm{BM}$, and $\mathrm{HZ}$ are from Genecast Biotechnology Co., Ltd. The authors have no other conflicts of interest to declare.

Ethics Statement: The authors are accountable for all aspects of the work in ensuring that questions related to the accuracy or integrity of any part of the work are appropriately investigated and resolved. This study was 


\section{Page 12 of 13}

conducted in accordance with the Declaration of Helsinki (as revised in 2013). This study was approved by the Beijing Cancer Hospital Ethics Committee (No. 2017KT91) and individual consent for this retrospective analysis was waived.

Open Access Statement: This is an Open Access article distributed in accordance with the Creative Commons Attribution-NonCommercial-NoDerivs 4.0 International License (CC BY-NC-ND 4.0), which permits the noncommercial replication and distribution of the article with the strict proviso that no changes or edits are made and the original work is properly cited (including links to both the formal publication through the relevant DOI and the license). See: https://creativecommons.org/licenses/by-nc-nd/4.0/.

\section{References}

1. Siegel RL, Miller KD, Jemal A. Cancer statistics, 2018. CA Cancer J Clin 2018;68:7-30.

2. Qiu M, Yang D, Cosgrove D, et al. Pattern of distant metastases in colorectal cancer: a SEER based study. Oncotarget 2015;6:38658-66.

3. Li J, Yuan Y, Yang F, et al. Expert consensus on multidisciplinary therapy of colorectal cancer with lung metastases (2019 edition). J Hematol Oncol 2019;12:16.

4. Tampellini M, Ottone A, Bellini E, et al. The role of lung metastasis resection in improving outcome of colorectal cancer patients: results from a large retrospective study. Oncologist 2012;17:1430-8.

5. Wang Z, Wang X, Yuan J, et al. Survival Benefit of Palliative Local Treatments and Efficacy of Different Pharmacotherapies in Colorectal Cancer With Lung Metastasis: Results From a Large Retrospective Study. Clin Colorectal Cancer 2018;17:e233-55.

6. Imanishi M, Yamamoto Y, Hamano Y, et al. Efficacy of adjuvant chemotherapy after resection of pulmonary metastasis from colorectal cancer: a propensity scorematched analysis. Eur J Cancer 2019;106:69-77.

7. Li WH. Oncological outcome of unresectable lung metastases without extrapulmonary metastases in colorectal cancer. World J Gastroenterol 2010;16:3318.

8. Nozawa H, Ishihara S, Kawai K, et al. Characterization of Conversion Chemotherapy for Secondary Surgical Resection in Colorectal Cancer Patients with Lung Metastases. Oncology 2017;92:135-41.

9. Prasanna T, Craft PS, Chua YJ, et al. The outcome of patients (pts) with metastatic colorectal cancer (mCRC) based on site of metastases (mets) and the impact of
Wang et al. Genetic features of lung and liver metastases in CRC

molecular markers and site of primary cancer on metastatic pattern. J Clin Oncol 2017;35:abstr 3560.

10. El-Deiry WS, Vijayvergia N, Xiu J, et al. Molecular profiling of 6,892 colorectal cancer samples suggests different possible treatment options specific to metastatic sites. Cancer Biol Ther 2015;16:1726-37.

11. Sartore-Bianchi A, Trusolino L, Martino C, et al. Dualtargeted therapy with trastuzumab and lapatinib in treatment-refractory, KRAS codon 12/13 wild-type, HER2-positive metastatic colorectal cancer (HERACLES): a proof-of-concept, multicentre, open-label, phase 2 trial. Lancet Oncol 2016;17:738-46.

12. Hainsworth JD, Meric-Bernstam F, Swanton C, et al. Targeted Therapy for Advanced Solid Tumors on the Basis of Molecular Profiles: Results From MyPathway, an OpenLabel, Phase IIa Multiple Basket Study. J Clin Oncol 2018;36:536-42.

13. Kovaleva V, Geissler AL, Lutz L, et al. Spatio-temporal mutation profiles of case-matched colorectal carcinomas and their metastases reveal unique de novo mutations in metachronous lung metastases by targeted next generation sequencing. Mol Cancer 2016;15:63.

14. Hou J, Zhang Y, Zhu Z. Gene heterogeneity in metastasis of colorectal cancer to the lung. Semin Cell Dev Biol 2017;64:58-64.

15. Sanchez-Vega F, Mina M, Armenia J, et al. Oncogenic Signaling Pathways in The Cancer Genome Atlas. Cell 2018;173:321-37.e10.

16. Chiang JM, Hsieh PS, Chen JS, et al. Rectal cancer level significantly affects rates and patterns of distant metastases among rectal cancer patients post curative-intent surgery without neoadjuvant therapy. World J Surg Oncol 2014;12:197.

17. He WZ, Hu WM, Wang F, et al. Comparison of Mismatch Repair Status Between Primary and Matched Metastatic Sites in Patients With Colorectal Cancer. J Natl Compr Canc Netw 2019;17:1174-83.

18. Cejas P, López-Gómez M, Aguayo C, et al. KRAS mutations in primary colorectal cancer tumors and related metastases: a potential role in prediction of lung metastasis. PLoS One 2009;4:e8199.

19. Kim MJ, Lee H, Kim JH, et al. Different metastatic pattern according to the KRAS mutational status and site-specific discordance of KRAS status in patients with colorectal cancer. BMC Cancer 2012;12:347.

20. Kemeny NE, Chou JF, Capanu M, et al. KRAS mutation influences recurrence patterns in patients undergoing hepatic resection of colorectal metastases. Cancer 
2014; 120:3965-71.

21. Morris VK, Lucas FA, Overman MJ, et al. Clinicopathologic characteristics and gene expression analyses of non-KRAS 12/13, RAS-mutated metastatic colorectal cancer. Ann Oncol 2014;25:2008-14.

22. Russo AL, Borger DR, Szymonifka J, et al. Mutational analysis and clinical correlation of metastatic colorectal cancer. Cancer 2014;120:1482-90.

23. Casimiro S, Fernandes A, Oliveira AG, et al. Metadherin expression and lung relapse in patients with colorectal carcinoma. Clin Exp Metastasis 2014;31:689-96.

24. Chudnovsky Y, Kim D, Zheng S, et al. ZFHX4 interacts with the NuRD core member CHD4 and regulates the glioblastoma tumor-initiating cell state. Cell Rep 2014;6:313-24.

25. Xu C. Exploring genetic mechanism of ZHFX4 in colorectal cancer by genome analysis. Shenyang: Dalian Medical University, 2016.

26. Xu K, Wang J, Gao J, et al. GATA binding protein 2 overexpression is associated with poor prognosis in KRAS mutant colorectal cancer. Oncol Rep 2016;36:1672-8.

27. Lao VV, Welcsh P, Luo Y, et al. Altered RECQ Helicase Expression in Sporadic Primary Colorectal Cancers. Transl Oncol 2013;6:458-69.

28. Liu Z, Wang P, Chen H, et al. Drug Discovery Targeting Bromodomain-Containing Protein 4. J Med Chem 2017;60:4533-58.

29. Hu Y, Zhou J, Ye F, et al. BRD4 inhibitor inhibits colorectal cancer growth and metastasis. Int J Mol Sci 2015;16:1928-48.

30. Xu C, Ooi WF, Qamra A, et al. HNF4 $\alpha$ pathway mapping identifies wild-type IDH1 as a targetable metabolic node in gastric cancer. Gut 2020;69:231-42.

31. Schwartz B, Algamas-Dimantov A, Hertz R, et al. Inhibition of colorectal cancer by targeting hepatocyte nuclear factor-4alpha. Int J Cancer 2009;124:1081-9.

32. Rimassa L, Bozzarelli S, Pietrantonio F, et al. Phase II Study of Tivantinib and Cetuximab in Patients With KRAS Wild-type Metastatic Colorectal Cancer With Acquired Resistance to EGFR Inhibitors and Emergence

Cite this article as: Wang Z, Zheng X, Wang X, Chen Y, Li Z, Yu J, Yang W, Mao B, Zhang H, Li J, Shen L. Genetic differences between lung metastases and liver metastases from left-sided microsatellite stable colorectal cancer: next generation sequencing and clinical implications. Ann Transl Med 2021;9(12):967. doi: 10.21037/atm-21-2221 of MET Overexpression: Lesson Learned for Future Trials With EGFR/MET Dual Inhibition. Clin Colorectal Cancer 2019;18:125-32.e2.

33. Bardelli A, Corso S, Bertotti A, et al. Amplification of the MET receptor drives resistance to anti-EGFR therapies in colorectal cancer. Cancer Discov 2013;3:658-73.

34. Yaeger R, Chatila WK, Lipsyc MD, et al. Clinical Sequencing Defines the Genomic Landscape of Metastatic Colorectal Cancer. Cancer Cell 2018;33:125-36.

35. Govindan R, Fakih MG, Price TJ, et al. 446PD - Phase I study of AMG 510, a novel molecule targeting KRAS G12C mutant solid tumours. Ann Oncol 2019;30:v163-4.

36. Harvey KF, Zhang X, Thomas DM. The Hippo pathway and human cancer. Nat Rev Cancer 2013;13:246-57.

37. Chen EX, Jonker DJ, Loree JM, et al. CCTG CO.26: Updated analysis and impact of plasma-detected microsatellite stability (MSS) and tumor mutation burden (TMB) in a phase II trial of durvalumab (D) plus tremelimumab (T) and best supportive care (BSC) versus BSC alone in patients (pts) with refractory metastatic colorectal carcinoma (rmCRC). J Clin Oncol 2019;37:abstr 3512 .

38. Fukuoka S, Hara H, Takahashi N, et al. Regorafenib plus nivolumab in patients with advanced gastric (GC) or colorectal cancer (CRC): An open-label, dose-finding, and dose-expansion phase $1 \mathrm{~b}$ trial (REGONIVO, EPOC1603). J Clin Oncol 2020;38:2053-61.

39. Shitara K, Hara H, Takahashi N, et al. Updated results from a phase $\mathrm{Ib}$ trial of regorafenib plus nivolumab in patients with advanced colorectal or gastric cancer (REGONIVO, EPOC1603). J Clin Oncol 2020;38:abstr135.

40. Tang W, Ren L, Liu T, et al. Bevacizumab Plus mFOLFOX6 Versus mFOLFOX6 Alone as First-Line Treatment for RAS Mutant Unresectable Colorectal Liver-Limited Metastases: The BECOME Randomized Controlled Trial. J Clin Oncol 2020;38:3175-84.

(English Language Editor: C. Betlazar-Maseh) 


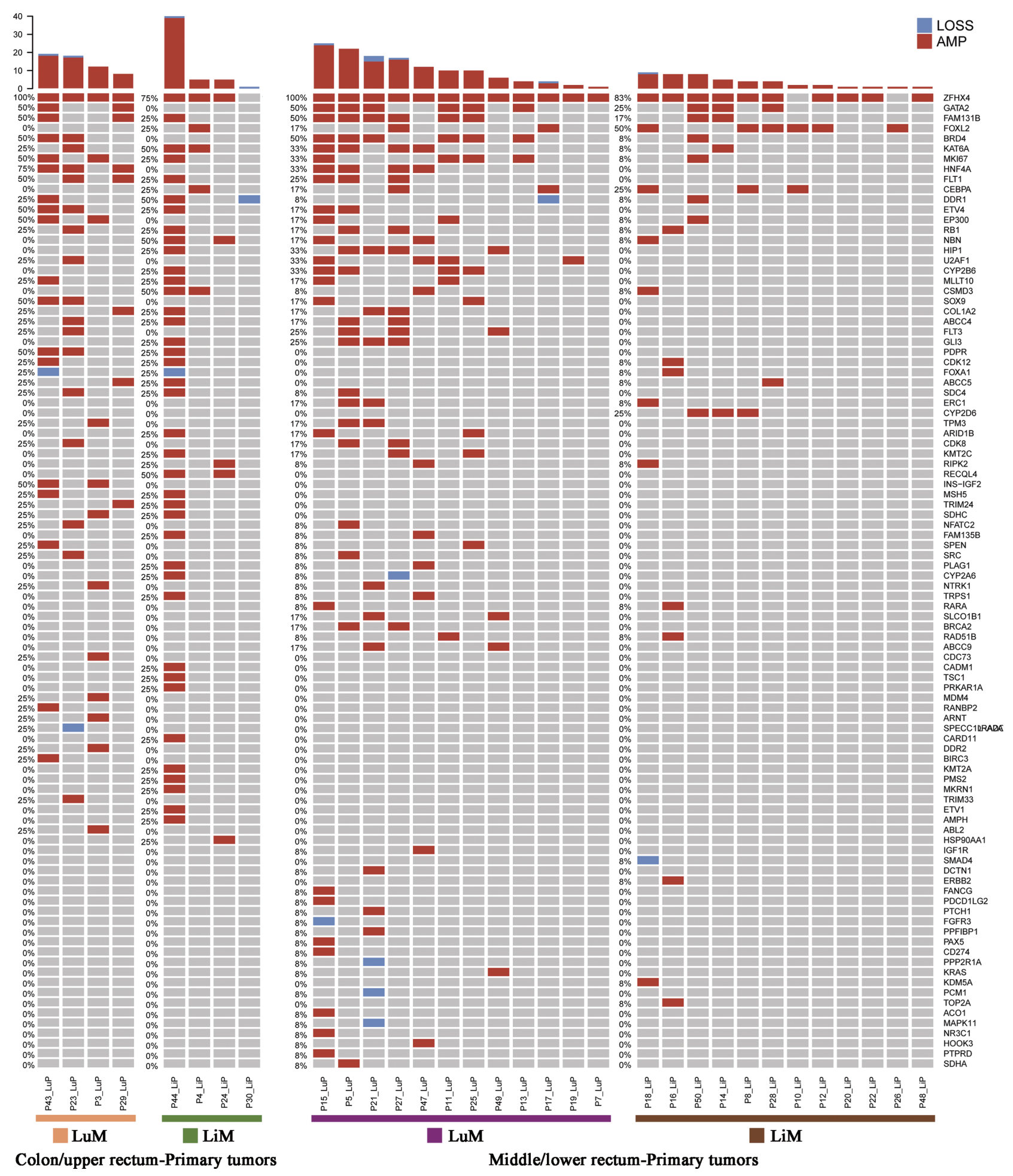

Figure S1 Copy number alterations at different locations in the primary tumors from the LuM and LiM cohorts. LuM, lung metastases; LiM, liver metastases; AMP, amplification. 
Table S1 Single nucleotide variations in the primary tumors from the LuM, LiM and control cohorts, n (\%)

\begin{tabular}{|c|c|c|c|c|c|c|}
\hline \multirow{2}{*}{ Gene } & LuM + LiM & Control & $P$ & LuM & LiM & $P$ \\
\hline & $n=32$ & $n=10$ & & $n=16$ & $n=16$ & \\
\hline TP53 & $20(62.5)$ & $9(90.0)$ & 0.211 & $11(68.8)$ & $9(56.3)$ & 0.716 \\
\hline$A P C$ & $17(53.1)$ & 8 (80.0) & 0.253 & $9(56.3)$ & $10(62.5)$ & 1.000 \\
\hline KRAS & $8(25.0)$ & $2(20.0)$ & 1.000 & $6(37.5)$ & 5 (31.3) & 1.000 \\
\hline FBXW7 & 5 (15.6) & $2(20.0)$ & 1.000 & $3(18.8)$ & 2 (12.5) & 1.000 \\
\hline PIКЗCA & 5 (15.6) & $1(10.0)$ & 1.000 & $4(25.0)$ & $0(0.0)$ & 0.101 \\
\hline$A L K$ & $3(9.4)$ & $0(0.0)$ & 1.000 & 3 (18.8) & 2 (12.5) & 1.000 \\
\hline ERBB2 & $3(9.4)$ & $0(0.0)$ & 1.000 & $3(18.8)$ & $1(6.3)$ & 0.600 \\
\hline ERBB3 & 4 (12.5) & $1(10.0)$ & 1.000 & $3(18.8)$ & $0(0.0)$ & 0.226 \\
\hline RYR2 & $3(9.4)$ & $0(0.0)$ & 1.000 & $3(18.8)$ & $1(6.3)$ & 0.600 \\
\hline$I D H 1$ & $1(3.1)$ & $0(0.0)$ & 1.000 & $1(6.3)$ & $2(12.5)$ & 1.000 \\
\hline HRAS & $2(6.3)$ & $1(10.0)$ & 1.000 & $1(6.3)$ & $1(6.3)$ & 1.000 \\
\hline TSPYL2 & $3(9.4)$ & $0(0.0)$ & 1.000 & $3(18.8)$ & $0(0.0)$ & 0.226 \\
\hline$D S T$ & $2(6.3)$ & $1(10.0)$ & 1.000 & $1(6.3)$ & $1(6.3)$ & 1.000 \\
\hline GNAS & $3(9.4)$ & $0(0.0)$ & 1.000 & $3(18.8)$ & $0(0.0)$ & 0.226 \\
\hline SMAD4 & $3(9.4)$ & $2(20.0)$ & 0.729 & $1(6.3)$ & $0(0.0)$ & 1.000 \\
\hline NTRK1 & $0(0.0)$ & $0(0.0)$ & - & $0(0.0)$ & $3(18.8)$ & 0.226 \\
\hline LGALS3 & $3(9.4)$ & $0(0.0)$ & 1.000 & $3(18.8)$ & $0(0.0)$ & 0.226 \\
\hline EGFR & $2(6.3)$ & $0(0.0)$ & 1.000 & 2 (12.5) & $1(6.3)$ & 1.000 \\
\hline$B R D 7$ & $2(6.3)$ & $0(0.0)$ & 1.000 & 2 (12.5) & $1(6.3)$ & 1.000 \\
\hline EIF3A & $3(9.4)$ & $0(0.0)$ & 1.000 & 3 (18.8) & $0(0.0)$ & 0.226 \\
\hline AMER1 & $2(6.3)$ & $1(10.0)$ & 1.000 & $1(6.3)$ & $1(6.3)$ & 1.000 \\
\hline RNF43 & $2(6.3)$ & $0(0.0)$ & 1.000 & 2 (12.5) & $1(6.3)$ & 1.000 \\
\hline MEF2B & $1(3.1)$ & $0(0.0)$ & 1.000 & $1(6.3)$ & 2 (12.5) & 1.000 \\
\hline TET2 & $2(6.3)$ & $0(0.0)$ & 1.000 & 2 (12.5) & $1(6.3)$ & 1.000 \\
\hline NRAS & $2(6.3)$ & 1 (10.0) & 1.000 & $1(6.3)$ & $1(6.3)$ & 1.000 \\
\hline PRKCB & $2(6.3)$ & $0(0.0)$ & 1.000 & 2 (12.5) & $1(6.3)$ & 1.000 \\
\hline PFKFB1 & $3(9.4)$ & $0(0.0)$ & 1.000 & $3(18.8)$ & $0(0.0)$ & 0.226 \\
\hline
\end{tabular}

LuM, lung metastases; LiM, liver metastases. 
Table S2 Copy number variations in the primary tumors from the LuM, LiM and control cohorts, $\mathrm{n}(\%)$

\begin{tabular}{|c|c|c|c|c|c|c|}
\hline Gene & LuM + LiM (n=32) & Control $(n=10)$ & $\mathrm{P}$ & LuM $(n=16)$ & $\operatorname{LiM}(n=16)$ & $P$ \\
\hline ZFHX4 & 29 (90.6) & $1(10.0)$ & $<0.001^{\#}$ & $16(100.0)$ & $13(81.3)$ & 0.226 \\
\hline GATA2 & $11(34.4)$ & $0(0.0)$ & 0.081 & $8(50.0)$ & $3(18.8)$ & 0.135 \\
\hline FAM131B & $11(34.4)$ & $0(0.0)$ & 0.081 & $8(50.0)$ & $3(18.8)$ & 0.135 \\
\hline HNF4A & 7 (21.9) & $4(40.0)$ & 0.468 & $7(43.8)$ & $0(0.0)$ & $0.007^{\#}$ \\
\hline RECQL4 & $2(6.3)$ & $7(70.0)$ & $<0.001^{\#}$ & $0(0.0)$ & $2(12.5)$ & 0.484 \\
\hline FOXL2 & $9(28.1)$ & $0(0.0)$ & 0.147 & $2(12.5)$ & $7(43.8)$ & 0.113 \\
\hline$B R D 4$ & $9(28.1)$ & $0(0.0)$ & 0.147 & $8(50.0)$ & $1(6.3)$ & $0.015^{\#}$ \\
\hline CEBPA & $6(18.8)$ & $3(30.0)$ & 0.753 & $2(12.5)$ & $4(25.0)$ & 0.654 \\
\hline KAT6A & $8(25.0)$ & $0(0.0)$ & 0.195 & $5(31.3)$ & $3(18.8)$ & 0.685 \\
\hline MKI67 & $8(25.0)$ & $0(0.0)$ & 0.195 & $6(37.5)$ & $2(12.5)$ & 0.220 \\
\hline$F L T 1$ & 6 (18.8) & $2(20.0)$ & 1.000 & 5 (31.3) & $1(6.3)$ & 0.172 \\
\hline$D D R 1$ * & 5 (15.6) & $2(20.0)$ & 1.000 & $2(12.5)$ & 3 (18.8) & 1.000 \\
\hline$N B N$ & 5 (15.6) & $2(20.0)$ & 1.000 & $2(12.5)$ & 3 (18.8) & 1.000 \\
\hline RIPK2 & $3(9.4)$ & $3(30.0)$ & 0.267 & $1(6.3)$ & $2(12.5)$ & 1.000 \\
\hline$R B 1$ & 5 (15.6) & $1(10.0)$ & 1.000 & 3 (18.8) & $2(12.5)$ & 1.000 \\
\hline CYP2A6 * & $2(6.3)$ & $3(30.0)$ & 0.143 & $1(6.3)$ & $1(6.3)$ & 1.000 \\
\hline HIP1 & $5(15.6)$ & $0(0.0)$ & 0.440 & $4(25.0)$ & $1(6.3)$ & 0.333 \\
\hline U2AF1 & 5 (15.6) & $0(0.0)$ & 0.440 & 5 (31.3) & $0(0.0)$ & $0.043^{\#}$ \\
\hline EP300 & 5 (15.6) & $0(0.0)$ & 0.440 & $4(25.0)$ & $1(6.3)$ & 0.333 \\
\hline ETV4 & 5 (15.6) & $0(0.0)$ & 0.440 & $4(25.0)$ & $1(6.3)$ & 0.333 \\
\hline CYP2B6 & 5 (15.6) & $0(0.0)$ & 0.440 & $4(25.0)$ & $1(6.3)$ & 0.333 \\
\hline$C D K 8$ & $3(9.4)$ & $2(20.0)$ & 0.729 & $3(18.8)$ & $0(0.0)$ & 0.226 \\
\hline SDC4 & $3(9.4)$ & $2(20.0)$ & 0.729 & $2(12.5)$ & $1(6.3)$ & 1.000 \\
\hline CSMD3 & $4(12.5)$ & $1(10.0)$ & 1.000 & $1(6.3)$ & 3 (18.8) & 0.600 \\
\hline$A B C C 4$ & $4(12.5)$ & $1(10.0)$ & 1.000 & $3(18.8)$ & $1(6.3)$ & 0.600 \\
\hline GL/3 & $4(12.5)$ & $1(10.0)$ & 1.000 & 3 (18.8) & $1(6.3)$ & 0.600 \\
\hline NFATC2 & $2(6.3)$ & $2(20.0)$ & 0.236 & $2(12.5)$ & $0(0.0)$ & 0.484 \\
\hline$S R C$ & $2(6.3)$ & $2(20.0)$ & 0.236 & $2(12.5)$ & $0(0.0)$ & 0.484 \\
\hline TRPS1 & $2(6.3)$ & $2(20.0)$ & 0.236 & $1(6.3)$ & $1(6.3)$ & 1.000 \\
\hline MLLT10 & $4(12.5)$ & $0(0.0)$ & 0.557 & $3(18.8)$ & $1(6.3)$ & 0.600 \\
\hline FLT3 & 4 (12.5) & $0(0.0)$ & 0.557 & $4(25.0)$ & $0(0.0)$ & 0.101 \\
\hline sox9 & 4 (12.5) & $0(0.0)$ & 0.557 & $4(25.0)$ & $0(0.0)$ & 0.101 \\
\hline COL1A2 & $4(12.5)$ & $0(0.0)$ & 0.557 & 3 (18.8) & $1(6.3)$ & 0.600 \\
\hline CYP2D6 & $3(9.4)$ & $1(10.0)$ & 1.000 & $0(0.0)$ & 3 (18.8) & 0.226 \\
\hline$P D P R$ & $3(9.4)$ & $1(10.0)$ & 1.000 & $2(12.5)$ & $1(6.3)$ & 1.000 \\
\hline$F L C N^{\&}$ & $0(0.0)$ & $3(30.0)$ & $0.010^{\#}$ & $0(0.0)$ & $0(0.0)$ & / \\
\hline ETV1 & $1(3.1)$ & $2(20.0)$ & 0.136 & $0(0.0)$ & $1(6.3)$ & 1.000 \\
\hline TPM3 & $3(9.4)$ & $0(0.0)$ & 1.000 & 3 (18.8) & $0(0.0)$ & 0.226 \\
\hline$E R C 1$ & $3(9.4)$ & $0(0.0)$ & 1.000 & 2 (12.5) & $1(6.3)$ & 1.000 \\
\hline$C D K 12$ & $3(9.4)$ & $0(0.0)$ & 1.000 & $1(6.3)$ & $2(12.5)$ & 1.000 \\
\hline$A R I D 1 B$ & $3(9.4)$ & $0(0.0)$ & 1.000 & $2(12.5)$ & $1(6.3)$ & 1.000 \\
\hline FOXA1* & $3(9.4)$ & $0(0.0)$ & 1.000 & $1(6.3)$ & $2(12.5)$ & 1.000 \\
\hline FAM135B & $2(6.3)$ & $1(10.0)$ & 1.000 & $1(6.3)$ & $1(6.3)$ & 1.000 \\
\hline BRCA2 & $2(6.3)$ & $1(10.0)$ & 1.000 & $2(12.5)$ & $0(0.0)$ & 0.484 \\
\hline PLAG1 & $2(6.3)$ & $1(10.0)$ & 1.000 & $1(6.3)$ & $1(6.3)$ & 1.000 \\
\hline KMT2C & $3(9.4)$ & $0(0.0)$ & 1.000 & $2(12.5)$ & $1(6.3)$ & 1.000 \\
\hline$A B C C 5$ & $3(9.4)$ & $0(0.0)$ & 1.000 & $1(6.3)$ & $2(12.5)$ & 1.000 \\
\hline
\end{tabular}

*, CNVs included both loss and amplification; ${ }^{*}$, only the FLCN gene showed a loss of copy number. Other genes showed only amplification; ${ }^{\text {, }}$ P values $<0.05$. LuM, lung metastases; LiM, liver metastases. 
Table S3 Alteration frequencies of colorectal cancer pathways and related genes in the primary tumors and metastatic lesions

\begin{tabular}{|c|c|c|c|c|c|c|c|c|c|}
\hline \multirow[b]{2}{*}{ Pathway } & \multirow[b]{2}{*}{ Gene } & \multicolumn{5}{|c|}{ Primary tumors, n (\%) } & \multicolumn{3}{|c|}{ Metastatic lesions, n (\%) } \\
\hline & & $\begin{array}{l}\text { LuM cohort } \\
\qquad(n=16)\end{array}$ & $\begin{array}{l}\text { LiM cohort } \\
\quad(n=16)\end{array}$ & $\begin{array}{l}\text { Control cohort } \\
\qquad(n=10)\end{array}$ & $P_{\text {LuM vs. LiM }}$ & $P_{\text {LuM vs. Control }}$ & $\begin{array}{l}\text { LuM cohort } \\
\qquad(n=15)\end{array}$ & $\begin{array}{l}\text { LiM cohort } \\
\quad(n=18)\end{array}$ & $P$ \\
\hline \multirow[t]{8}{*}{ WNT } & & $10(62.5)$ & $11(68.8)$ & $8(80.0)$ & 1.000 & 0.420 & $9(60.0)$ & 13 (72.2) & 0.488 \\
\hline & $A P C$ & $9(56.3)$ & $10(62.5)$ & $8(80.0)$ & 1.000 & 0.399 & $8(53.3)$ & $12(66.7)$ & 0.493 \\
\hline & AMER1 & $1(6.3)$ & $1(6.3)$ & $1(10.0)$ & 1.000 & 1.000 & $0(0.0)$ & $1(5.6)$ & 1.000 \\
\hline & RNF43 & $2(12.5)$ & $1(6.3)$ & $0(0.0)$ & 1.000 & 0.508 & $1(6.7)$ & $0(0.0)$ & 0.455 \\
\hline & CTNNB1 & $1(6.3)$ & $0(0.0)$ & $0(0.0)$ & 1.000 & 1.000 & $1(6.7)$ & $0(0.0)$ & 0.455 \\
\hline & GSK3B & $0(0.0)$ & $0(0.0)$ & $0(0.0)$ & - & - & $1(6.7)$ & $1(5.6)$ & 1.000 \\
\hline & SFRP2 & $0(0.0)$ & $0(0.0)$ & $0(0.0)$ & - & - & $0(0.0)$ & $1(5.6)$ & 1.000 \\
\hline & TCF7L2 & $0(0.0)$ & $0(0.0)$ & $1(10.0)$ & - & 0.385 & $0(0.0)$ & $0(0.0)$ & - \\
\hline \multirow[t]{7}{*}{ TGF $\beta$} & & $2(12.5)$ & $3(18.8)$ & $3(30.0)$ & 1.000 & 0.340 & $4(26.7)$ & $4(22.2)$ & 1.000 \\
\hline & SMAD4 & $1(6.3)$ & $1(6.3)$ & $2(20.0)$ & 1.000 & 0.538 & $3(20.0)$ & $1(5.6)$ & 0.308 \\
\hline & SMAD2 & $0(0.0)$ & $0(0.0)$ & $1(10.0)$ & - & 0.385 & $1(6.7)$ & $2(11.1)$ & 1.000 \\
\hline & SMAD3 & $1(6.3)$ & $1(6.3)$ & $0(0.0)$ & 1.000 & 1.000 & $0(0.0)$ & $0(0.0)$ & - \\
\hline & TGFBR1 & $0(0.0)$ & $0(0.0)$ & $0(0.0)$ & - & - & $0(0.0)$ & $2(11.1)$ & 0.489 \\
\hline & ACVR1B & $0(0.0)$ & $0(0.0)$ & $0(0.0)$ & - & - & $1(6.7)$ & $0(0.0)$ & 0.455 \\
\hline & TGFBR2 & $0(0.0)$ & $1(6.3)$ & $0(0.0)$ & 1.000 & - & $0(0.0)$ & $0(0.0)$ & - \\
\hline \multirow[t]{30}{*}{ RTK/RAS } & & $14(87.5)$ & $11(68.8)$ & 7 (70.0) & 0.394 & 0.340 & 15 (100.0) & 13 (72.2) & $0.049^{\#}$ \\
\hline & KRAS & 7 (43.8) & 5 (31.3) & $3(30.0)$ & 0.716 & 0.683 & 11 (73.3) & 7 (38.9) & 0.080 \\
\hline & EGFR & 2 (12.5) & $1(6.3)$ & $1(10.0)$ & 1.000 & 1.000 & $1(6.7)$ & $4(22.2)$ & 0.346 \\
\hline & ERBB3 & 3 (18.8) & $0(0.0)$ & $1(10.0)$ & 0.226 & 1.000 & 2 (13.3) & $3(16.7)$ & 1.000 \\
\hline & NTRK1 & 2 (12.5) & $3(18.8)$ & $0(0.0)$ & 1.000 & 0.508 & 2 (13.3) & $2(11.1)$ & 1.000 \\
\hline & HRAS & $1(6.3)$ & $1(6.3)$ & $2(20.0)$ & 1.000 & 0.538 & $3(20.0)$ & $1(5.6)$ & 0.308 \\
\hline & $A L K$ & 3 (18.8) & 2 (12.5) & $0(0.0)$ & 1.000 & 0.262 & $0(0.0)$ & $2(11.1)$ & 0.489 \\
\hline & MET & $0(0.0)$ & $0(0.0)$ & $0(0.0)$ & - & - & $6(40.0)$ & $1(5.6)$ & $0.030^{\#}$ \\
\hline & ERBB2 & 3 (18.8) & $1(6.3)$ & $0(0.0)$ & 0.600 & 0.262 & $1(6.7)$ & $1(5.6)$ & 1.000 \\
\hline & FLT3 & $4(25.0)$ & $0(0.0)$ & $0(0.0)$ & 0.101 & 0.136 & $0(0.0)$ & $2(11.1)$ & 0.489 \\
\hline & $K I T$ & 2 (12.5) & $0(0.0)$ & $0(0.0)$ & 0.484 & 0.508 & 2 (13.3) & $2(11.1)$ & 1.000 \\
\hline & NRAS & $1(6.3)$ & $1(6.3)$ & $1(10.0)$ & 1.000 & 1.000 & $1(6.7)$ & $1(5.6)$ & 1.000 \\
\hline & FGFR3 & $2(12.5)$ & $0(0.0)$ & $0(0.0)$ & 0.484 & 0.508 & $1(6.7)$ & $1(5.6)$ & 1.000 \\
\hline & $B R A F$ & $1(6.3)$ & $0(0.0)$ & $0(0.0)$ & 1.000 & 1.000 & $1(6.7)$ & $1(5.6)$ & 1.000 \\
\hline & ERBB4 & $1(6.3)$ & $0(0.0)$ & $1(10.0)$ & 1.000 & 1.000 & $0(0.0)$ & $1(5.6)$ & 1.000 \\
\hline & FGFR2 & $0(0.0)$ & $0(0.0)$ & $0(0.0)$ & - & - & $1(6.7)$ & $2(11.1)$ & 1.000 \\
\hline & MAP2K1 & 2 (12.5) & $0(0.0)$ & $0(0.0)$ & 0.484 & 0.508 & $1(6.7)$ & $0(0.0)$ & 0.455 \\
\hline & NTRK3 & $1(6.3)$ & $0(0.0)$ & $1(10.0)$ & 1.000 & 1.000 & $1(6.7)$ & $0(0.0)$ & 0.455 \\
\hline & PDGFRA & 2 (12.5) & $0(0.0)$ & $0(0.0)$ & 0.484 & 0.508 & $1(6.7)$ & $0(0.0)$ & 0.455 \\
\hline & $C B L$ & $0(0.0)$ & $0(0.0)$ & $0(0.0)$ & - & - & $1(6.7)$ & $1(5.6)$ & 1.000 \\
\hline & NF1 & $0(0.0)$ & $0(0.0)$ & $0(0.0)$ & - & - & 2 (13.3) & $0(0.0)$ & 0.199 \\
\hline & PTPN11 & $0(0.0)$ & $0(0.0)$ & $0(0.0)$ & - & - & $1(6.7)$ & $1(5.6)$ & 1.000 \\
\hline & $R E T$ & $1(6.3)$ & $0(0.0)$ & $0(0.0)$ & 1.000 & 1.000 & $1(6.7)$ & $0(0.0)$ & 0.455 \\
\hline & $A R A F$ & $0(0.0)$ & $0(0.0)$ & $0(0.0)$ & - & - & $1(6.7)$ & $0(0.0)$ & 0.455 \\
\hline & FGFR1 & $0(0.0)$ & $0(0.0)$ & $0(0.0)$ & - & - & $0(0.0)$ & $1(5.6)$ & 1.000 \\
\hline & FGFR4 & $1(6.3)$ & $0(0.0)$ & $0(0.0)$ & 1.000 & 1.000 & $0(0.0)$ & $0(0.0)$ & - \\
\hline & IGF1R & $1(6.3)$ & $0(0.0)$ & $0(0.0)$ & 1.000 & 1.000 & $0(0.0)$ & $0(0.0)$ & - \\
\hline & MAP2K2 & $0(0.0)$ & $0(0.0)$ & $0(0.0)$ & - & - & $1(6.7)$ & $0(0.0)$ & 0.455 \\
\hline & MAPK1 & $1(6.3)$ & $0(0.0)$ & $0(0.0)$ & 1.000 & 1.000 & $0(0.0)$ & $0(0.0)$ & - \\
\hline & ROS1 & $0(0.0)$ & $0(0.0)$ & 1 (10.0) & - & 0.385 & $0(0.0)$ & $0(0.0)$ & - \\
\hline \multirow[t]{3}{*}{ PI3K } & & $8(50.0)$ & $4(25.0)$ & $2(20.0)$ & 0.273 & 0.218 & 8 (53.3) & 7 (38.9) & 0.494 \\
\hline & PIКЗCA & $4(25.0)$ & $0(0.0)$ & $1(10.0)$ & 0.101 & 0.617 & $1(6.7)$ & $3(16.7)$ & 0.607 \\
\hline & MTOR & $2(12.5)$ & $0(0.0)$ & $0(0.0)$ & 0.484 & 0.508 & $0(0.0)$ & $3(16.7)$ & 0.233 \\
\hline
\end{tabular}

Table S3 (continued) 


\begin{tabular}{|c|c|c|c|c|c|c|c|c|c|}
\hline \multirow[b]{2}{*}{ Pathway } & \multirow[b]{2}{*}{ Gene } & \multicolumn{5}{|c|}{ Primary tumors, n (\%) } & \multicolumn{3}{|c|}{ Metastatic lesions, n (\%) } \\
\hline & & $\begin{array}{l}\text { LuM cohort } \\
\qquad(n=16)\end{array}$ & $\begin{array}{l}\text { LiM cohort } \\
\quad(n=16)\end{array}$ & $\begin{array}{l}\text { Control cohort } \\
\qquad(n=10)\end{array}$ & $P_{\text {LuM vs. LiM }}$ & $P_{\text {LuM vs. Control }}$ & $\begin{array}{l}\text { LuM cohort } \\
\qquad(\mathrm{n}=15)\end{array}$ & $\begin{array}{l}\text { LiM cohort } \\
\qquad(n=18)\end{array}$ & $\mathrm{P}$ \\
\hline & STK11 & $1(6.3)$ & $0(0.0)$ & $1(10.0)$ & 1.000 & 1.000 & $2(13.3)$ & $0(0.0)$ & 0.199 \\
\hline & TSC1 & $0(0.0)$ & $1(6.3)$ & $0(0.0)$ & 1.000 & - & $1(6.7)$ & $2(11.1)$ & 1.000 \\
\hline & $P P P 2 R 1 A$ & $1(6.3)$ & $0(0.0)$ & $0(0.0)$ & 1.000 & 1.000 & $2(13.3)$ & $0(0.0)$ & 0.199 \\
\hline & PTEN & $1(6.3)$ & $1(6.3)$ & $0(0.0)$ & 1.000 & 1.000 & $0(0.0)$ & $1(5.6)$ & 1.000 \\
\hline & $A K T 1$ & $0(0.0)$ & $0(0.0)$ & $0(0.0)$ & - & - & $2(13.3)$ & $0(0.0)$ & 0.199 \\
\hline & AKT3 & $2(12.5)$ & $0(0.0)$ & $0(0.0)$ & 0.484 & 0.508 & $0(0.0)$ & $0(0.0)$ & - \\
\hline & PIKЗCB & $1(6.3)$ & $0(0.0)$ & $0(0.0)$ & 1.000 & 1.000 & $1(6.7)$ & $0(0.0)$ & 0.455 \\
\hline & PIK3R1 & $0(0.0)$ & $1(6.3)$ & $0(0.0)$ & 1.000 & - & $0(0.0)$ & $1(5.6)$ & 1.000 \\
\hline & TSC2 & $1(6.3)$ & $0(0.0)$ & $0(0.0)$ & 1.000 & 1.000 & $1(6.7)$ & $0(0.0)$ & 0.455 \\
\hline & PIK3R2 & $0(0.0)$ & $0(0.0)$ & $0(0.0)$ & - & - & $1(6.7)$ & $0(0.0)$ & 0.455 \\
\hline & RICTOR & $0(0.0)$ & $1(6.3)$ & $0(0.0)$ & 1.000 & - & $0(0.0)$ & $0(0.0)$ & - \\
\hline \multirow[t]{5}{*}{ P53 } & & $12(75.0)$ & $10(62.5)$ & $9(90.0)$ & 0.704 & 0.617 & 14 (93.3) & $12(66.7)$ & 0.095 \\
\hline & TP53 & $11(68.8)$ & 9 (56.3) & $9(90.0)$ & 0.716 & 0.352 & 13 (86.7) & $12(66.7)$ & 0.242 \\
\hline & MDM4 & 2 (12.5) & $0(0.0)$ & $0(0.0)$ & 0.484 & 0.508 & 2 (13.3) & $0(0.0)$ & 0.199 \\
\hline & ATM & $0(0.0)$ & $0(0.0)$ & $0(0.0)$ & - & - & $1(6.7)$ & $0(0.0)$ & 0.455 \\
\hline & RPS6KA3 & $0(0.0)$ & $1(6.3)$ & $0(0.0)$ & 1.000 & - & $0(0.0)$ & $0(0.0)$ & - \\
\hline \multirow[t]{3}{*}{ NRF2 } & & $1(6.3)$ & $1(6.3)$ & $0(0.0)$ & 1.000 & 1.000 & $0(0.0)$ & $0(0.0)$ & - \\
\hline & CUL3 & $0(0.0)$ & $1(6.3)$ & $0(0.0)$ & 1.000 & - & $0(0.0)$ & $0(0.0)$ & - \\
\hline & KEAP1 & $1(6.3)$ & $0(0.0)$ & $0(0.0)$ & 1.000 & 1.000 & $0(0.0)$ & $0(0.0)$ & - \\
\hline \multirow[t]{9}{*}{ NOTCH } & & 9 (56.3) & 6 (37.5) & $3(30.0)$ & 0.479 & 0.248 & $7(46.7)$ & $8(44.4)$ & 1.000 \\
\hline & FBXW7 & 3 (18.8) & 2 (12.5) & $2(20.0)$ & 1.000 & 1.000 & 2 (13.3) & $3(16.7)$ & 1.000 \\
\hline & EP300 & $4(25.0)$ & $1(6.3)$ & $0(0.0)$ & 0.333 & 0.136 & 2 (13.3) & 2 (11.1) & 1.000 \\
\hline & KDM5A & $0(0.0)$ & $1(6.3)$ & $0(0.0)$ & 1.000 & - & $3(20.0)$ & $3(16.7)$ & 1.000 \\
\hline & NOTCH1 & $1(6.3)$ & $1(6.3)$ & 1 (10.0) & 1.000 & 1.000 & 2 (13.3) & $0(0.0)$ & 0.199 \\
\hline & NOTCH2 & $1(6.3)$ & $0(0.0)$ & $0(0.0)$ & 1.000 & 1.000 & $1(6.7)$ & 2 (11.1) & 1.000 \\
\hline & NOTCH3 & $1(6.3)$ & $1(6.3)$ & $0(0.0)$ & 1.000 & 1.000 & $0(0.0)$ & $0(0.0)$ & - \\
\hline & SPEN & 2 (12.5) & $0(0.0)$ & $0(0.0)$ & 0.484 & 0.508 & $0(0.0)$ & $0(0.0)$ & - \\
\hline & NCOR1 & $1(6.3)$ & $0(0.0)$ & $0(0.0)$ & 1.000 & 1.000 & $0(0.0)$ & $0(0.0)$ & - \\
\hline \multirow[t]{4}{*}{ MYC } & & $1(6.3)$ & $0(0.0)$ & $0(0.0)$ & 1.000 & 1.000 & 2 (13.3) & $0(0.0)$ & 0.199 \\
\hline & $M A X$ & $0(0.0)$ & $0(0.0)$ & $0(0.0)$ & - & - & $1(6.7)$ & $0(0.0)$ & 0.455 \\
\hline & $M Y C L$ & $1(6.3)$ & $0(0.0)$ & $0(0.0)$ & 1.000 & 1.000 & $0(0.0)$ & $0(0.0)$ & - \\
\hline & MYCN & $0(0.0)$ & $0(0.0)$ & $0(0.0)$ & - & - & $1(6.7)$ & $0(0.0)$ & 0.455 \\
\hline \multirow[t]{6}{*}{ HIPPO } & & 2 (12.5) & 2 (12.5) & $0(0.0)$ & 1.000 & 0.508 & 5 (33.3) & $1(5.6)$ & 0.070 \\
\hline & FAT3 & $0(0.0)$ & $1(6.3)$ & $0(0.0)$ & 1.000 & - & $3(20.0)$ & $0(0.0)$ & 0.083 \\
\hline & FAT4 & $0(0.0)$ & $0(0.0)$ & $0(0.0)$ & - & - & 2 (13.3) & $1(5.6)$ & 0.579 \\
\hline & FAT1 & $0(0.0)$ & $0(0.0)$ & $0(0.0)$ & - & - & $1(6.7)$ & 1 (5.6) & 1.000 \\
\hline & FAT2 & $1(6.3)$ & $1(6.3)$ & $0(0.0)$ & 1.000 & 1.000 & $0(0.0)$ & $0(0.0)$ & - \\
\hline & NF2 & $1(6.3)$ & $0(0.0)$ & $0(0.0)$ & 1.000 & 1.000 & $0(0.0)$ & $0(0.0)$ & - \\
\hline \multirow[t]{9}{*}{ Cell cycle } & & 5 (31.3) & 3 (18.8) & $2(20.0)$ & 0.685 & 0.668 & $4(26.7)$ & 5 (27.8) & 1.000 \\
\hline & $R B 1$ & $4(25.0)$ & 3 (18.8) & $1(10.0)$ & 1.000 & 0.617 & 2 (13.3) & $3(16.7)$ & 1.000 \\
\hline & $C D K 4$ & $0(0.0)$ & $0(0.0)$ & $1(10.0)$ & - & 0.385 & 2 (13.3) & $0(0.0)$ & 0.199 \\
\hline & CCND3 & $0(0.0)$ & $0(0.0)$ & $0(0.0)$ & - & - & $0(0.0)$ & $1(5.6)$ & 1.000 \\
\hline & CCNE1 & $0(0.0)$ & $0(0.0)$ & $0(0.0)$ & - & - & $1(6.7)$ & $0(0.0)$ & 0.455 \\
\hline & $C D K N 1 A$ & $0(0.0)$ & $0(0.0)$ & $0(0.0)$ & - & - & $1(6.7)$ & $0(0.0)$ & 0.455 \\
\hline & $C D K N 2 C$ & $0(0.0)$ & $0(0.0)$ & $0(0.0)$ & - & - & $1(6.7)$ & $0(0.0)$ & 0.455 \\
\hline & $E 2 F 1$ & $1(6.3)$ & $0(0.0)$ & $0(0.0)$ & 1.000 & 1.000 & $0(0.0)$ & $0(0.0)$ & - \\
\hline & CDKN2A & $0(0.0)$ & $0(0.0)$ & $0(0.0)$ & - & - & $0(0.0)$ & 1 (5.6) & 1.000 \\
\hline
\end{tabular}

\footnotetext{
" $\mathrm{P}$ values $<0.05$. LuM, lung metastases; LiM, liver metastases.
} 
Table S4 Comparison of the frequencies of possible gene alterations associated with immune checkpoint inhibitors

\begin{tabular}{|c|c|c|c|c|c|c|c|c|c|c|c|}
\hline \multirow[b]{2}{*}{ Gene alteration } & \multicolumn{5}{|c|}{ Cohort (n) } & \multicolumn{6}{|c|}{$\mathrm{P}$} \\
\hline & $\begin{array}{l}\text { LuM-P } \\
(n=16)\end{array}$ & $\begin{array}{l}\text { LiM-P } \\
(n=16)\end{array}$ & $\begin{array}{l}\text { Control-P } \\
\quad(n=10)\end{array}$ & $\begin{array}{l}\text { LuM-M } \\
(n=15)\end{array}$ & $\begin{array}{l}\text { LiM-M } \\
(n=18)\end{array}$ & $\begin{array}{c}\text { LuM-P vs. } \\
\text { LiM-P }\end{array}$ & $\begin{array}{l}\text { LuM-P vs. } \\
\text { Control-P }\end{array}$ & $\begin{array}{l}\text { LiM-P vs. } \\
\text { Control-P }\end{array}$ & $\begin{array}{l}\text { LuM-M vs. } \\
\text { LiM-M }\end{array}$ & $\begin{array}{l}\text { LuM-P vs. } \\
\text { LuM-M }\end{array}$ & $\begin{array}{c}\text { LiM-P vs. } \\
\text { LiM-M }\end{array}$ \\
\hline \multicolumn{12}{|l|}{ TP53 } \\
\hline \multicolumn{12}{|l|}{ Mutation } \\
\hline+ & 11 & 9 & 9 & 13 & 12 & 0.716 & 0.352 & 0.099 & 0.242 & 0.394 & 0.725 \\
\hline- & 5 & 7 & 1 & 2 & 6 & & & & & & \\
\hline \multicolumn{12}{|l|}{ KRAS } \\
\hline \multicolumn{12}{|l|}{ Mutation } \\
\hline+ & 6 & 5 & 2 & 9 & 7 & 1 & 0.42 & 0.668 & 0.303 & 0.289 & 0.729 \\
\hline - & 10 & 11 & 8 & 6 & 11 & & & & & & \\
\hline \multicolumn{12}{|l|}{ NRAS } \\
\hline \multicolumn{12}{|l|}{ Mutation } \\
\hline+ & 1 & 1 & 1 & 1 & 1 & 1 & 1 & 1 & 1 & 1 & 1 \\
\hline- & 15 & 15 & 9 & 14 & 17 & & & & & & \\
\hline \multicolumn{12}{|l|}{ ERCC2 } \\
\hline \multicolumn{12}{|l|}{ Mutation } \\
\hline+ & 0 & 2 & 0 & 3 & 0 & 0.484 & - & 0.508 & 0.083 & 0.101 & 0.214 \\
\hline- & 16 & 14 & 10 & 12 & 18 & & & & & & \\
\hline \multicolumn{12}{|l|}{ POLE } \\
\hline \multicolumn{12}{|l|}{ Loss } \\
\hline+ & 0 & 0 & 1 & 0 & 0 & - & 0.385 & 0.385 & - & - & - \\
\hline - & 16 & 16 & 9 & 15 & 18 & & & & & & \\
\hline \multicolumn{12}{|l|}{ Mutation } \\
\hline+ & 1 & 1 & 0 & 1 & 0 & 1 & 1 & 1 & 0.455 & 1 & 0.471 \\
\hline- & 15 & 15 & 10 & 14 & 18 & & & & & & \\
\hline \multicolumn{12}{|l|}{$M L H 1$} \\
\hline \multicolumn{12}{|l|}{ Mutation } \\
\hline+ & 1 & 1 & 0 & 1 & 1 & 1 & 1 & 1 & 1 & 1 & 1 \\
\hline- & 15 & 15 & 10 & 14 & 17 & & & & & & \\
\hline \multicolumn{12}{|l|}{ PMS2 } \\
\hline \multicolumn{12}{|l|}{ Mutation } \\
\hline+ & 2 & 0 & 0 & 1 & 0 & 0.484 & 0.508 & - & 0.455 & 1 & - \\
\hline- & 14 & 16 & 10 & 14 & 18 & & & & & & \\
\hline \multicolumn{12}{|l|}{ MSH6 } \\
\hline Mutation & & & & & & & & & & & \\
\hline+ & 2 & 0 & 0 & 0 & 0 & 0.484 & 0.508 & - & - & 0.484 & - \\
\hline- & 14 & 16 & 10 & 15 & 18 & & & & & & \\
\hline BRCA2 & & & & & & & & & & & \\
\hline Mutation & & & & & & & & & & & \\
\hline+ & 0 & 0 & 0 & 1 & 1 & - & - & - & 1 & 1 & 1 \\
\hline- & 16 & 16 & 10 & 14 & 17 & & & & & & \\
\hline MDM4 & & & & & & & & & & & \\
\hline Gain & & & & & & & & & & & \\
\hline+ & 1 & 0 & 0 & 1 & 0 & 1 & 1 & - & 0.455 & 1 & - \\
\hline- & 15 & 16 & 10 & 14 & 18 & & & & & & \\
\hline PBRM1 & & & & & & & & & & & \\
\hline Mutation & & & & & & & & & & & \\
\hline+ & 1 & 0 & 0 & 0 & 0 & 1 & 1 & - & - & 1 & - \\
\hline - & 15 & 16 & 10 & 15 & 18 & & & & & & \\
\hline
\end{tabular}

Table S4 (continued) 


\begin{tabular}{|c|c|c|c|c|c|c|c|c|c|c|c|}
\hline \multirow[b]{2}{*}{ Gene alteration } & \multicolumn{5}{|c|}{ Cohort (n) } & \multicolumn{6}{|c|}{$\mathrm{P}$} \\
\hline & $\begin{array}{l}\text { LuM-P } \\
(n=16)\end{array}$ & $\begin{array}{l}\text { LiM-P } \\
(n=16)\end{array}$ & $\begin{array}{l}\text { Control-P } \\
(n=10)\end{array}$ & $\begin{array}{l}\text { LuM-M } \\
(n=15)\end{array}$ & $\begin{array}{l}\text { LiM-M } \\
(n=18)\end{array}$ & $\begin{array}{l}\text { LuM-P vs. } \\
\text { LiM-P }\end{array}$ & $\begin{array}{l}\text { LuM-P vs. } \\
\text { Control-P }\end{array}$ & $\begin{array}{l}\text { LiM-P vs. } \\
\text { Control-P }\end{array}$ & $\begin{array}{l}\text { LuM-M vs. } \\
\text { LiM-M }\end{array}$ & $\begin{array}{c}\text { LuM-P vs. } \\
\text { LuM-M }\end{array}$ & $\begin{array}{c}\text { LiM-P vs. } \\
\text { LiM-M }\end{array}$ \\
\hline \multicolumn{12}{|l|}{$B R C A 1$} \\
\hline \multicolumn{12}{|l|}{ Mutation } \\
\hline+ & 0 & 0 & 0 & 0 & 1 & - & - & - & 1 & - & 1 \\
\hline- & 16 & 16 & 10 & 15 & 17 & & & & & & \\
\hline \multicolumn{12}{|l|}{ ATM } \\
\hline \multicolumn{12}{|l|}{ Mutation } \\
\hline+ & 0 & 0 & 0 & 1 & 0 & - & - & - & 0.455 & 1 & - \\
\hline- & 16 & 16 & 10 & 14 & 18 & & & & & & \\
\hline \multicolumn{12}{|l|}{$E G F R$} \\
\hline \multicolumn{12}{|l|}{ Gain } \\
\hline+ & 0 & 0 & 1 & 1 & 1 & - & 0.385 & 0.385 & 1 & 0.484 & 1 \\
\hline- & 16 & 16 & 9 & 14 & 17 & & & & & & \\
\hline \multicolumn{12}{|l|}{ Mutation } \\
\hline+ & 2 & 1 & 0 & 0 & 3 & 1 & 0.508 & 1 & 0.233 & 0.484 & 0.604 \\
\hline- & 14 & 15 & 10 & 15 & 15 & & & & & & \\
\hline \multicolumn{12}{|l|}{ STK11 } \\
\hline \multicolumn{12}{|l|}{ Mutation } \\
\hline+ & 1 & 0 & 0 & 2 & 0 & 1 & 1 & - & 0.199 & 0.6 & - \\
\hline- & 15 & 16 & 10 & 13 & 18 & & & & & & \\
\hline \multicolumn{12}{|l|}{ MET } \\
\hline \multicolumn{12}{|l|}{ Mutation } \\
\hline+ & 0 & 0 & 0 & 2 & 1 & - & - & - & 0.579 & 0.226 & 1 \\
\hline- & 16 & 16 & 10 & 13 & 17 & & & & & & \\
\hline \multicolumn{12}{|l|}{ CTNNB1 } \\
\hline \multicolumn{12}{|l|}{ Mutation } \\
\hline+ & 1 & 0 & 0 & 1 & 0 & 1 & 1 & - & 0.455 & 1 & - \\
\hline- & 15 & 16 & 10 & 14 & 18 & & & & & & \\
\hline \multicolumn{12}{|l|}{$J A K 1$} \\
\hline \multicolumn{12}{|l|}{ Mutation } \\
\hline+ & 0 & 0 & 0 & 1 & 0 & - & - & - & 0.455 & 1 & - \\
\hline- & 16 & 16 & 10 & 14 & 18 & & & & & & \\
\hline \multicolumn{12}{|l|}{ PTEN } \\
\hline \multicolumn{12}{|l|}{ Loss } \\
\hline+ & 0 & 0 & 0 & 0 & 1 & - & - & - & 1 & - & 1 \\
\hline- & 16 & 16 & 10 & 15 & 17 & & & & & & \\
\hline
\end{tabular}

LuM, lung metastases; LiM, liver metastases; P, primary tumor; $M$, metastatic lesion. 